\title{
IRREDUCIBLE GAUGE THEORIES IN THE FRAMEWORK OF THE Sp(2)-COVARIANT QUANTIZATION METHOD
}

\author{
P. M. LAVROV' \\ Tomsk State Pedagogical Institute, Tomsk, 634041, Russia \\ P. YU. MOSHIN and A. A. RESHETNYAK \\ Tomsk State University, Tomsk, 634050, Russia
}

\begin{abstract}
Irreducible gauge theories in both the Lagrangian and Hamiltonian versions of the $\mathrm{Sp}(2)-$ covariant quantization method are studied. Solutions to generating equations are obtained in the form of expansions in power series of ghost and auxiliary variables up to the $3 \mathrm{~d}$ order inclusively.
\end{abstract}

\section{Introduction}

The advanced quantization methods for gauge theories in both the Lagrangian $^{1-3}$ and Hamiltonian ${ }^{4-6}$ formalisms are based on the idea of a special type of global supersymetry, the so-called BRST (Becchi - Rouet - Stora - Tyutin) symmetry. ${ }^{7-8}$ It turns out, however, that the BRST invariance requirement for a theory may be strengthened by a requirement of extended BRST invariance. The extended BRST symmetry transformations (have been discussed in part in Refs. 9-12) include both the BRST and anti-BRST transformations (in the Yang - Mills theories the anti-BRST symmetry has been introduced in Refs. 13-14) with the fermion parameters of the BRST and anti-BRST transformations to form a nature doublet under the global symplectic group $\operatorname{Sp}(2)$ (see for example Ref. 12).

The quantization rules based on the extended BRST symmetry principle for general gauge theories in both the Lagrangian and Hamiltonian formalisms (the $\operatorname{Sp}(2)$-covariant quantization method) have been recently proposed. ${ }^{15-20}$ Namely, in Refs. $15-17$ an $\operatorname{Sp}(2)$-covariant formulation of the 
BV (Batalin - Vilkovisky) Lagrangian quantization method ${ }^{2,3}$ for general gauge theories of any stage reducibility has been developed. In its turn, the corresponding Hamiltonian version, being the $\mathrm{Sp}(2)$-covariant formulation of the BFV (Batalin - Fradkin - Vilkovisky) generalized canonical quantization method ${ }^{4-6}$ for arbitrary any-stage reducible dynamical systems with both first- and second-class constraints, has been suggested in Refs. 18-20. Note in this connection that for the first time the BFV method has been applied to an analysis of the extended BRST symmetry in Ref. 11. It should be also pointed out that in Ref. 12 in the case of dynamical systems subject to first-class constraints with constant structural coeffitients, there has been obtained the unitarizing Hamiltonian ${ }^{4-6}$ invariant under the extended BRST symmetry transformations for an arbitrary choice of the gauge.

The global symplectic group $\operatorname{Sp}(2)$ providing a basis for the quantization rules $^{15-20}$ plays the key role in the formalism proposed and demonstrates the advantages it yields. That is to say, in Refs. 16, 19 it is shown that ghost and auxiliary variables for any-stage reducible field theory in both the Lagrangian (fields of the total configuration spase) and Hamiltonian (generalized momenta and coordinates) versions form components of completely symmetric tensors under the group $\operatorname{Sp}(2)$.

It is noteworthy that the sets of canonical variables in the framework of both the standard ${ }^{4-6}$ and $\mathrm{Sp}(2)$-covariant ${ }^{18-20}$ Hamiltonian formulations coincide. Meanwhile, the set of variables (specifically, the one of antifields) in the $\mathrm{Sp}(2)$-covariant version ${ }^{15-17}$ of the Lagrangian quantization method is redundant with respect to the set of variables in the standard version. ${ }^{2,3}$

The basic objects in the Hamiltonian version of the $\operatorname{Sp}(2)$-covariant quantization method are the boson function $\mathcal{H}$ and the doublet of fermion functions $\Omega^{a}{ }^{18-20}$ while the Lagrangian version is based on the boson functional $S{ }^{15-17}$ The objects concerned satisfy gauge algebra generating equations ${ }^{15-20}$ and permit constructing the quantum action of a theory. Proof of the existence theorems for solutions to the gauge algebra generating equations of 
both the Lagrangian ${ }^{15-17}$ and Hamiltonian ${ }^{18-20}$ versions as well as description of arbitrariness in the solutions are given in Refs. 15, 17, 18, 20.

In this paper we shall restrict ourselves to consideration of irreducible gauge theories only. Namely, there are studied arbitrary dynamical systems with lineary independent first-class constraints in the Hamiltonian version $^{18-20}$ and general gauge theories with lineary independent generators of gauge transformations in the Lagrangian version. ${ }^{15-17}$ The explicit solutions to the generating equations ${ }^{15-20}$ are obtained in the form of expansions in power series of ghost and auxiliary variables up to the third order inclusively. It is shown that in the $\mathrm{Sp}(2)$-covariant formulation ${ }^{15-20}$ of both the BV and BFV quantization methods, solutions to the generating equations are completely defined by the gauge algebra structural relations as they are in the standard formulation. ${ }^{2-6}$

Note, from the perturbation theory viewpoint, that the solutions obtained are quite sufficient for all practical purposes of the field theory. Indeed, an application of the perturbation theory implies one's knowledge of propagators and interaction vertices. The propagators are defined by kernels of differential operators present in the kinetic part of the quantum action. One has, therefore, apart from the initial classical action, to have at one's disposal the first approximation for the quantum action. The interaction vertices, in turn, are defined by the higher approximations (along with the above-mentioned ones) for solutions to the generating equations. Since the majority of the field theory calculations are at best the two-loop ones, the approximations up to the $3 \mathrm{~d}$ order turn out to provide such calculations. Moreover, in the number of gauge theories with a closed algebra, the iterations concerned yield, in both the Lagrangian and Hamiltonian versions, an exact form for the quantum action. It is these considerations that made us seek solutions to the generating equations of the $\operatorname{Sp}(2)$-covariant quantization method with an accuracy up to the $3 \mathrm{~d}$ order in ghost and auxiliary variables. 
In this paper we use the condensed notations ${ }^{21}$ and designations of Refs. 1520. The derivatives with respect to generalized momenta or antifields are always understood as left and those with respect to the corresponding configuration space variables (i. e. generalized coordinates $Q^{A}$ or fields $\phi^{A}$ ) as right unless specified. The left derivatives with respect to $Q^{A}$ or $\phi^{A}$ are labelled "l": $\delta_{\mathrm{l}} / \delta Q^{A}, \delta_{\mathrm{l}} / \delta \phi^{A}$. The Grassmann parity of a certain quantity $A$ is denoted $\varepsilon(A)$ and the new ghost number ${ }^{15-20}-\operatorname{ngh}(A)$.

We make use of the standard definition ${ }^{22}$ of the Poisson superbracket in an extended phase space $\Gamma=\left(P_{A}, Q^{A}\right)$

$$
\{F, G\}=\frac{\delta F}{\delta Q^{A}} \frac{\delta G}{\delta P_{A}}-\frac{\delta G}{\delta Q^{A}} \frac{\delta F}{\delta P_{A}}(-1)^{\varepsilon(F) \varepsilon(G)},
$$

where $P_{A}$ are the set of generalized momenta congugate to coordinates $Q^{A}$

$$
\varepsilon\left(P_{A}\right)=\varepsilon\left(Q^{A}\right), \quad \operatorname{ngh}\left(P_{A}\right)=-\operatorname{ngh}\left(Q^{A}\right) .
$$

The superbracket (1.1) possesses the standart algebraic properties among which we only point out the Jacobi identity

$$
\{\{F, G\}, H\}(-1)^{\varepsilon(F) \varepsilon(H)}+\text { cycl.perm. }(F G H) \equiv 0 \text {. }
$$

The indices of the global symplectic group $\operatorname{Sp}(2)$ are denoted $a, b, c, \ldots$, and assume two values $a=1,2$. The invariant tensor of the group $\operatorname{Sp}(2)$, being a constant antisymmetric tensor of rank two, is denoted $\varepsilon^{a b}$, such that $\varepsilon^{12}=1$. Symmetrization over the $\operatorname{Sp}(2)$ indices is understood in the form

$$
A^{\{a b\}}=A^{a b}+A^{b a} \text {. }
$$

\section{The Solution of Equations for $\mathcal{H}, \Omega^{a}$}

It may be convinient to remind the reader about the key points of the Hamiltonian version of the $\operatorname{Sp}(2)$-covariant quantization method. ${ }^{18-20}$ To do this, consider the dynamical system described in the phase spase

$$
\eta=\left(p_{\imath}, q^{\imath}\right), \quad \operatorname{ngh}\left(q^{\imath}\right)=0
$$


by the classical Hamiltonian $H_{0}=H_{0}(\eta)$ and by the set of lineary independent first-class constraints $T_{\alpha}=T_{\alpha}(\eta), \varepsilon\left(T_{\alpha}\right) \equiv \varepsilon_{\alpha}$ with the involution relations

$$
\left\{T_{\alpha}, T_{\beta}\right\}=T_{\gamma} U_{\alpha \beta}^{\gamma}, \quad\left\{H_{0}, T_{\alpha}\right\}=T_{\beta} V_{\alpha}^{\beta}
$$

where the structural coeffitients $U_{\alpha \beta}^{\gamma}$ possess the properties of generalized antisymmetry

$$
U_{\alpha \beta}^{\gamma}=-(-1)^{\varepsilon_{\alpha} \varepsilon_{\beta}} U_{\beta \alpha}^{\gamma}
$$

Given this, the structure of the extended phase space $\Gamma=\left(P_{A}, Q^{A}\right)$ is as follows ${ }^{18}$

$$
\Gamma=\left(P_{A}, Q^{A}\right)=\left(\eta ; \mathcal{P}_{\alpha a}, C^{\alpha a} ; \lambda_{\alpha}, \pi^{\alpha}\right)
$$

In (2.3) $C^{\alpha a}$ form $\mathrm{Sp}(2)$ doublets of ghost variables

$$
\varepsilon\left(C^{\alpha a}\right)=\varepsilon_{\alpha}+1, \quad \operatorname{ngh}\left(C^{\alpha a}\right)=1
$$

and $\pi^{\alpha}$ are auxiliary variables

$$
\varepsilon\left(\pi^{\alpha}\right)=\varepsilon_{\alpha}, \quad \operatorname{ngh}\left(\pi^{\alpha}\right)=2,
$$

introducing the gauge in the framework of the standard formulation of the generalized canonical quantization method.

The boson function $\mathcal{H}$ and the fermion functions $\Omega^{a}$ introduced in Ref. 18 satisfy the $\operatorname{Sp}(2)$-covariant generating equations

$$
\left\{\Omega^{a}, \Omega^{b}\right\}=0, \quad\left\{\mathcal{H}, \Omega^{a}\right\}=0,
$$

with the boundary conditions

$$
\left.\frac{\delta \Omega^{a}}{\delta C^{\alpha b}}\right|_{C=\pi=\mathcal{P}=\lambda=0}=T_{\alpha} \delta_{b}^{a},\left.\quad \frac{\delta \Omega^{a}}{\delta \pi^{\alpha}}\right|_{C=\pi=\lambda=0}=\varepsilon^{a b} \mathcal{P}_{\alpha b}
$$




$$
\left.\mathcal{H}\right|_{C=\pi=\mathcal{P}=\lambda=0}=H_{0}
$$

The total unitarizing Hamiltonian $H$ is now determined in terms of $\mathcal{H}$ and $\Omega^{a}$ by the formula ${ }^{18-20}$

$$
H=\mathcal{H}+\frac{1}{2} \varepsilon_{a b}\left\{\left\{\Phi, \Omega^{b}\right\}, \Omega^{b}\right\}
$$

where $\Phi$ is the boson function fixing a concrete choice of admissible gauge. An essential property of the unitarizing Haimltonian $H(2.6)$ is its invariance under the extended BRST transformations of the phase space $\Gamma$

$$
\delta \Gamma=\left\{\Gamma, \Omega^{a}\right\} \mu_{a} .
$$

Here $\mu_{a}$ is an $\mathrm{Sp}(2)$ doublet of constant Grassmann parameters of extended BRST symmetry. Owing to the properties of the functions $\Omega^{a}(2.4)$, the transformations (2.7) are nilpotent.

For the theory in question with the Hamiltonian $H(2.6)$, the generating functional of the Green's functions is given in the usual form by the functional integral ${ }^{18}$

$$
Z(I)=\int d \Gamma \exp \left\{\frac{i}{\hbar} \int d t\left(P_{A} \dot{Q}^{A}-H+I \Gamma\right)\right\} .
$$

As a consequence of invariance of the total Hamiltonian under the transformations (2.7), the vacuum functional $Z_{\Phi} \equiv Z(0)$ is independent ${ }^{18}$ on the choice of the gauge function $\Phi$. Indeed, one can readily establish that any change of the gauge $\Phi \rightarrow \Phi+\Delta \Phi$ in the functional integral (2.8) for $I=0$ can be compensated by the change of the integration variables $(2.7)$ $\Gamma \rightarrow \Gamma+\delta \Gamma$ with the parameters

$$
\mu_{a}=\frac{i}{2 \hbar} \varepsilon_{a b} \int d t\left\{\Omega^{b}, \Delta \Phi\right\}
$$

Hence $Z_{\Phi+\Delta \Phi}=Z_{\Phi}$, and therefore, the $S$ matrix is gauge invariant in the Hamiltonian version of the $\operatorname{Sp}(2)$-covariant quantization method. 
In Ref. 18 it is shown that one can seek solutions to Eqs. (2.4) in the form of expansions in power series of ghost $C^{\alpha a}$ and auxiliary $\pi^{\alpha}$ variables

$$
\Omega^{a}=\sum_{n=1}^{\infty} \Omega_{n}^{a}, \quad \mathcal{H}=H_{0}+\sum_{n=1}^{\infty} \mathcal{H}_{n}
$$

having required the Grassmann parity and the new ghost number to be conserved in every order of perturbation series

$$
\varepsilon\left(\Omega_{n}^{a}\right)=\operatorname{ngh}\left(\Omega_{n}^{a}\right)=1, \quad \varepsilon\left(\mathcal{H}_{n}\right)=\operatorname{ngh}\left(\mathcal{H}_{n}\right)=0 .
$$

In (2.9) $\Omega_{n}^{a}$ and $\mathcal{H}_{n}$ are some $n$th order polinomials in the variables $C^{\alpha a}$, $\pi^{\alpha}$. The requirement of the new ghost number conservation leads to the fact that $\Omega_{n}^{a}$ and $\mathcal{H}_{n}$ must be polinomials in $\mathcal{P}_{\alpha a}, \lambda_{\alpha}$ as well.

In the first order perturbation series, the solution to Eqs. (2.4), determining $\Omega^{a}$, can be chosen in the form ${ }^{18}$

$$
\Omega_{1}^{a}=T_{\alpha} C^{\alpha a}+\varepsilon^{a b} \mathcal{P}_{\alpha b} \pi^{\alpha}
$$

Then, the higher approximations in (2.9) are determined by the equations ${ }^{18}$

$$
\begin{gathered}
W^{a} \mathcal{H}_{n+1}=D_{n+1}^{a}, \quad n \geq 1, \\
W^{\{a} \Omega_{n+1}^{b\}}=-B_{n+1}^{a b}, \quad n \geq 1,
\end{gathered}
$$

where the operators $W^{a}$ are given by the formula

$$
W^{a}=T_{\alpha} \frac{\delta}{\delta P_{\alpha a}}+\varepsilon^{a b} \mathcal{P}_{\alpha b} \frac{\delta}{\delta \lambda_{\alpha}}+(-1)^{\varepsilon_{\alpha}} \varepsilon^{a b} \pi^{\alpha} \frac{\delta_{1}}{\delta C^{\alpha b}}
$$

and possess the properties

$$
W^{\{a} W^{b\}}=0
$$

The functions $D_{n+1}^{a}$ and $B_{n+1}^{a b}$ are constructed from $\Omega_{m+1}^{a}, \mathcal{H}_{m}^{a}, m \leq n$ by the rules ${ }^{18}$

$$
\begin{gathered}
B_{n+1}^{a b}=\left\{\Omega_{[n]}^{a}, \Omega_{[n]}^{b}\right\}_{n+1}, \\
D_{n+1}^{a}=\left\{\mathcal{H}_{[n]}, \Omega_{[n+1]}^{a}\right\}_{n+1},
\end{gathered}
$$


where

$$
\Omega_{[n]}^{a} \equiv \sum_{k=1}^{n} \Omega_{k}^{a}, \quad \mathcal{H}_{[n]} \equiv H_{0}+\sum_{k=1}^{n} \mathcal{H}_{k},
$$

the symbol $\{,\}_{k}$ denotes the $k$ th order for the superbracket $\{$,$\} in power$ series of the variables $C^{\alpha a}, \pi^{\alpha}$. The functions $B_{n+1}^{a b}$ and $D_{n+1}^{a}$ satisfy the equations

$$
\begin{gathered}
W^{a} B_{n+1}^{b c}+\text { cycl.perm. }(a b c)=0, \\
W^{\{a} D_{n+1}^{b\}}=0,
\end{gathered}
$$

being the compatibility conditions for Eqs. (2.11), (2.12).

Let us give the explicit solutions to Eqs. (2.11), (2.12) when $n=1,2$ in the case of dynamical systems with irreducible first-class constraints described by the properties (2.1). The explicit form of $\Omega_{1}^{a}(2.10)$, as it follows from (2.14), (2.15), enables us to obtain the solutions to Eqs. (2.11), (2.12) for $\mathcal{H}_{1}, \Omega_{2}^{a}$. Note to this end that the functions $D_{1}^{a}$ and $B_{2}^{a b}$ have the form

$$
\begin{gathered}
D_{1}^{a}=\left\{H_{0}, T_{\alpha}\right\} C^{\alpha a}, \\
B_{2}^{a b}=-\left\{T_{\beta}, T_{\alpha}\right\}(-1)^{\varepsilon_{\beta}} C^{\alpha a} C^{\beta b} .
\end{gathered}
$$

It is convinient, seeking the solution to Eqs. (2.12) with the right-hand side (2.17), to make use of the following decomposition of an $\operatorname{Sp}(2)$ tensor of rank two, constructed from $C^{\alpha a}$

$$
(-1)^{\varepsilon_{\beta}} C^{\alpha a} C^{\beta b}=T^{\alpha \beta\{a b\}}+\hat{T}^{\alpha \beta} \varepsilon^{a b},
$$

where the components of decomposition

$$
T^{\alpha \beta\{a b\}}=\frac{1}{2}(-1)^{\varepsilon_{\beta}} C^{\alpha\{a} C^{\beta b\}}, \quad \hat{T}^{\alpha \beta} \varepsilon^{a b}=-\frac{1}{2}(-1)^{\varepsilon_{\beta}} \varepsilon_{c d} C^{\alpha c} C^{\beta d} \varepsilon^{a b}
$$

form respectively the symmetric and antisymmetric tensors of rank two under the group $\operatorname{Sp}(2)$. The quantities $T^{\alpha \beta\{a b\}}$ and $\hat{T}^{\alpha \beta}$ possess the following properties of generalized (anti)symmetry

$$
T^{\alpha \beta\{a b\}}=-(-1)^{\varepsilon_{\alpha} \varepsilon_{\beta}} T^{\beta \alpha\{a b\}}, \quad \hat{T}^{\alpha \beta}=(-1)^{\varepsilon_{\alpha} \varepsilon_{\beta}} \hat{T}^{\beta \alpha}
$$


Then, with allowance made for the involution relations (2.1), we find from the definition (2.18) and the properties (2.2), (2.19) the following representations for $D_{1}^{a}, B_{2}^{a b}$

$$
\begin{gathered}
D_{1}^{a}=T_{\beta} V_{\alpha}^{\beta} C^{\alpha a}, \\
B_{2}^{a b}=-T_{\gamma} U_{\beta \alpha}^{\gamma} T^{\alpha \beta\{a b\}} .
\end{gathered}
$$

Solving Eqs. (2.11) with the right-hand side (2.20) is as follows. It is necessary to choose such a function $X_{1}$ as to produce all (or several) structures present in $D_{1}^{a}$ when the operators $W^{a}$ act upon it

$$
X_{1}=\mathcal{P}_{\beta a} V_{\alpha}^{\beta} C^{\alpha a} \text {. }
$$

Then, applying $W^{a}$ to $X_{1}$

$$
W^{a} X_{1}=T_{\beta} V_{\alpha}^{\beta} C^{\alpha a}-\mathcal{P}_{\beta b} V_{\alpha}^{\beta} \pi^{\alpha} \varepsilon^{a b}
$$

we find that the first summand in the right-hand side (2.22) and $D_{1}^{a}(2.20)$ coincide. One readily observes that the second summand in (2.22) is reproduced by applying $W^{a}$ to the function $X_{2}$ of the form

$$
X_{2}=\lambda_{\beta} V_{\alpha}^{\beta} \pi^{\alpha}
$$

namely

$$
W^{a} X_{2}=\mathcal{P}_{\beta b} V_{\alpha}^{\beta} \pi^{\alpha} \varepsilon^{a b}
$$

Given this, it follows from the obvious relation

$$
W^{a}\left(X_{1}+X_{2}\right)-D_{1}^{a}=0
$$

that one can choose the solution to Eqs. (2.11) with the right-hand side (2.20) in the form

$$
\mathcal{H}_{1}=X_{1}+X_{2}=\mathcal{P}_{\beta a} V_{\alpha}^{\beta} C^{\alpha a}+\lambda_{\beta} V_{\alpha}^{\beta} \pi^{\alpha} .
$$

The method of solving Eqs. (2.12) with the right-hand side (2.21) is quite analogus to the one considered above. To this end, it suffices to apply the operators $W^{a}$ to the functions of the form

$$
X_{1}^{a}=\frac{1}{2} \mathcal{P}_{\gamma b} U_{\beta \alpha}^{\gamma} T^{\alpha \beta\{a b\}}, \quad X_{2}^{a}=-\frac{1}{2} \lambda_{\gamma} U_{\beta \alpha}^{\gamma}(-1)^{\varepsilon_{\beta}} C^{\alpha a} \pi^{\beta}
$$


then

$$
\begin{gathered}
W^{\{a} X_{1}^{b\}}=T_{\gamma} U_{\beta \alpha}^{\gamma} T^{\alpha \beta\{a b\}}+\frac{1}{2} \mathcal{P}_{\gamma c} U_{\beta \alpha}^{\gamma}(-1)^{\varepsilon_{\beta}} C^{\alpha\{a} \varepsilon^{b\} c} \pi^{\beta} \\
W^{\{a} X_{2}^{b\}}=-\frac{1}{2} \mathcal{P}_{\gamma c} U_{\beta \alpha}^{\gamma}(-1)^{\varepsilon_{\beta}} C^{\alpha\{a} \varepsilon^{b\} c} \pi^{\beta} .
\end{gathered}
$$

In (2.24) we made allowance for the generalized antisymmetry property (2.2) of the gauge algebra structural coeffitients $U_{\alpha \beta}^{\gamma}$, and, as a consequence, for the identity

$$
U_{\alpha \beta}^{\gamma} \pi^{\beta} \pi^{\alpha} \equiv 0
$$

Then, making a comparison between $B_{2}^{a b}$ and $W^{\{a} X_{1}^{b\}}, W^{\{a} X_{2}^{b\}}$, we have

$$
W^{\{a} X_{1}^{b\}}+W^{\{a} X_{2}^{b\}}+B_{2}^{a b}=0 .
$$

Consequently, the solution to Eqs. (2.12) with the right-hand side (2.21) can be chosen in the form

$$
\Omega_{2}^{a}=X_{1}^{a}+X_{2}^{a}=\frac{1}{2} \mathcal{P}_{\gamma b} U_{\beta \alpha}^{\gamma} T^{\alpha \beta\{a b\}}-\frac{1}{2} \lambda_{\gamma} U_{\beta \alpha}^{\gamma}(-1)^{\varepsilon_{\beta}} C^{\alpha a} \pi^{\beta} .
$$

Now consider, taking into account the explicit form of the functions $\mathcal{H}_{[1]}$ (2.23) and $\Omega_{[2]}^{a}(2.10),(2.25)$, the solution of Eqs. (2.11), (2.12) for $\mathcal{H}_{2}, \Omega_{3}^{a}$. By virtue of $(2.14),(2.15)$, we find $D_{2}^{a}$ and $B_{3}^{a b}$ in the form

$$
\begin{aligned}
D_{2}^{a}= & \frac{1}{2} \mathcal{P}_{\gamma b}\left(\left\{H_{0}, U_{\beta \alpha}^{\gamma}\right\}+V_{\delta}^{\gamma} U_{\beta \alpha}^{\delta}-U_{\beta \delta}^{\gamma} V_{\alpha}^{\delta}+(-1)^{\varepsilon_{\alpha} \varepsilon_{\beta}} U_{\alpha \delta}^{\gamma} V_{\beta}^{\delta}-\left\{V_{\beta}^{\gamma}, T_{\alpha}\right\}\right. \\
& \left.+(-1)^{\varepsilon_{\alpha} \varepsilon_{\beta}}\left\{V_{\alpha}^{\gamma}, T_{\beta}\right\}\right) T^{\alpha \beta\{a b\}}-\mathcal{P}_{\gamma b}\left\{V_{\beta}^{\gamma}, T_{\alpha}\right\} \hat{T}^{\alpha \beta} \varepsilon^{a b} \\
& -\frac{1}{2} \lambda_{\gamma}\left(\left\{H_{0}, U_{\beta \alpha}^{\gamma}\right\}+V_{\delta}^{\gamma} U_{\beta \alpha}^{\delta}-U_{\beta \delta}^{\gamma} V_{\alpha}^{\delta}+(-1)^{\varepsilon_{\alpha} \varepsilon_{\beta}} U_{\alpha \delta}^{\gamma} V_{\beta}^{\delta}\right. \\
& \left.-2\left\{V_{\beta}^{\gamma}, T_{\alpha}\right\}\right)(-1)^{\varepsilon_{\beta}} C^{\alpha a} \pi^{\beta} \\
B_{3}^{a b}= & -\frac{1}{2} \mathcal{P}_{\rho c}\left(\frac{1}{2} U_{\gamma \delta}^{\rho} U_{\beta \alpha}^{\delta}(-1)^{\varepsilon_{\alpha} \varepsilon_{\gamma}}+\frac{1}{2} U_{\alpha \delta}^{\rho} U_{\gamma \beta}^{\delta}(-1)^{\varepsilon_{\alpha} \varepsilon_{\beta}}\right. \\
& \left.-\left\{U_{\gamma \beta}^{\rho}, T_{\alpha}\right\}(-1)^{\varepsilon_{\alpha} \varepsilon_{\gamma}}\right)(-1)^{\varepsilon_{\beta}+\varepsilon_{\alpha} \varepsilon_{\gamma}} C^{\alpha\{a} C^{\beta b\}} C^{\gamma c} \\
& -\frac{1}{2} \lambda_{\rho}\left(\frac{1}{2} U_{\gamma \delta}^{\rho} U_{\beta \alpha}^{\delta}(-1)^{\varepsilon_{\alpha} \varepsilon_{\gamma}}+\frac{1}{2} U_{\alpha \delta}^{\rho} U_{\gamma \beta}^{\delta}(-1)^{\varepsilon_{\alpha} \varepsilon_{\beta}}\right. \\
& \left.-\left\{U_{\gamma \beta}^{\rho}, T_{\alpha}\right\}(-1)^{\varepsilon_{\alpha} \varepsilon_{\gamma}}\right)(-1)^{\varepsilon_{\alpha} \varepsilon_{\gamma}} T^{\alpha \beta\{a b\}} \pi^{\gamma}
\end{aligned}
$$


here we made use of the definition (2.18) and the properties (2.19).

Let us now introduce, for the sake of convinience, the following decomposition of an $\mathrm{Sp}(2)$ tensor of rank three present in $B_{3}^{a b}$

$$
\begin{aligned}
\frac{1}{2}(-1)^{\varepsilon_{\beta}+\varepsilon_{\alpha} \varepsilon_{\beta}} C^{\alpha a} C^{\beta b} C^{\gamma c}= & \frac{1}{2} T^{\alpha \beta \gamma\{a b c\}} \\
& +\frac{1}{3} \varepsilon^{a b}\left(\hat{T}^{\alpha \beta} C^{\gamma c}(-1)^{\varepsilon_{\alpha} \varepsilon_{\gamma}}-\hat{T}^{\beta \gamma} C^{\alpha c}(-1)^{\varepsilon_{\alpha} \varepsilon_{\beta}}\right) \\
& +\frac{1}{3} \varepsilon^{a c}\left(\hat{T}^{\beta \gamma} C^{\alpha b}(-1)^{\varepsilon_{\alpha} \varepsilon_{\beta}}-\hat{T}^{\gamma \alpha} C^{\beta b}(-1)^{\varepsilon_{\beta} \varepsilon_{\gamma}}\right),
\end{aligned}
$$

where

$$
T^{\alpha \beta \gamma\{a b c\}}=\frac{1}{3}(-1)^{\varepsilon_{\alpha} \varepsilon_{\gamma}} T^{\alpha \beta\{a b\}} C^{\gamma c}+\text { cycl.perm. }(a b c)
$$

is a symmetric tensor of rank three under the group $\operatorname{Sp}(2)$. From the definition of the tensor (2.28) there follow the properties

$$
T^{\alpha \beta \gamma\{a b c\}}=-(-1)^{\varepsilon_{\alpha \beta \gamma}} T^{\beta \alpha \gamma\{a b c\}}=-(-1)^{\varepsilon_{\alpha \beta \gamma}} T^{\alpha \gamma \beta\{a b c\}},
$$

where

$$
\varepsilon_{\alpha \beta \gamma} \equiv \varepsilon_{\alpha} \varepsilon_{\beta}+\varepsilon_{\alpha} \varepsilon_{\gamma}+\varepsilon_{\beta} \varepsilon_{\gamma} .
$$

To solve Eqs. (2.11), (2.12) for $\mathcal{H}_{2}, \Omega_{3}^{a}$ it is necessary to make use of the gauge algebra structural relations, being the consequence of the involution relations (2.1), namely

$$
\begin{gathered}
T_{\delta}\left(\left\{H_{0}, U_{\alpha \beta}^{\delta}\right\}+V_{\gamma}^{\delta} U_{\alpha \beta}^{\gamma}-U_{\alpha \gamma}^{\delta} V_{\beta}^{\gamma}+(-1)^{\varepsilon_{\alpha} \varepsilon_{\beta}} U_{\beta \gamma}^{\delta} V_{\alpha}^{\gamma}\right. \\
\left.-\left\{V_{\alpha}^{\delta}, T_{\beta}\right\}+(-1)^{\varepsilon_{\alpha} \varepsilon_{\beta}}\left\{V_{\beta}^{\delta}, T_{\alpha}\right\}\right) \equiv 0, \\
T_{\rho}\left(\left\{U_{\alpha \beta}^{\rho}, T_{\gamma}\right\}-U_{\alpha \delta}^{\rho} U_{\beta \gamma}^{\delta}\right)(-1)^{\varepsilon_{\alpha} \varepsilon_{\gamma}}+\text { cycl.perm. }(\alpha \beta \gamma) \equiv 0 .
\end{gathered}
$$

The relations $(2.30),(2.31)$ follow respectively from the Jacobi identities (1.2) of the form

$$
\left\{\left\{T_{\alpha}, T_{\beta}\right\}, H_{0}\right\}+\left\{\left\{H_{0}, T_{\alpha}\right\}, T_{\beta}\right\}+\left\{\left\{T_{\beta}, H_{0}\right\}, T_{\alpha}\right\}(-1)^{\varepsilon_{\alpha} \varepsilon_{\beta}} \equiv 0
$$




$$
\left\{\left\{T_{\alpha}, T_{\beta}\right\}, T_{\gamma}\right\}(-1)^{\varepsilon_{\alpha} \varepsilon_{\gamma}}+\text { cycl.perm. }(\alpha \beta \gamma) \equiv 0 .
$$

Then, by virtue of lineary independence of the constraints $T_{\alpha}$, we conclude that there exist such structural coeffitients $E_{\alpha \beta}^{\gamma \delta}$ and $E_{\alpha \beta \gamma}^{\delta \rho}$ with the properties

$$
\begin{gathered}
E_{\alpha \beta}^{\gamma \delta}=-(-1)^{\varepsilon_{\alpha} \varepsilon_{\beta}} E_{\beta \alpha}^{\gamma \delta}=-(-1)^{\varepsilon_{\gamma} \varepsilon_{\delta}} E_{\alpha \beta}^{\delta \gamma}, \\
E_{\alpha \beta \gamma}^{\delta \rho}=-(-1)^{\varepsilon_{\rho} \varepsilon_{\delta}} E_{\alpha \beta \gamma}^{\rho \delta}, \quad E_{\alpha \beta \gamma}^{\delta \rho}=E_{\gamma \alpha \beta}^{\delta \rho},
\end{gathered}
$$

that the relations $(2.30),(2.31)$ could be represented in the form

$$
\begin{aligned}
& T_{\gamma} E_{\alpha \beta}^{\gamma \delta}= \frac{1}{2}\left(\left\{H_{0}, U_{\alpha \beta}^{\delta}\right\}+V_{\gamma}^{\delta} U_{\alpha \beta}^{\gamma}-U_{\alpha \gamma}^{\delta} V_{\beta}^{\gamma}+(-1)^{\varepsilon_{\alpha} \varepsilon_{\beta}} U_{\beta \gamma}^{\delta} V_{\alpha}^{\gamma}\right. \\
&\left.-\left\{V_{\alpha}^{\delta}, T_{\beta}\right\}+(-1)^{\varepsilon_{\alpha} \varepsilon_{\beta}}\left\{V_{\beta}^{\delta}, T_{\alpha}\right\}\right), \\
& T_{\delta} E_{\alpha \beta \gamma}^{\delta \rho}=\frac{1}{3}\left(U_{\alpha \delta}^{\rho} U_{\beta \gamma}^{\delta}-\left\{U_{\alpha \beta}^{\rho}, T_{\gamma}\right\}\right)(-1)^{\varepsilon_{\alpha} \varepsilon_{\gamma}}+\text { cycl.perm. }(\alpha \beta \gamma) .
\end{aligned}
$$

From (2.33), (2.34) with allowance made for the properties (2.29), there follow the representations for the functions $D_{2}^{a}$ and $B_{3}^{a b}$

$$
\begin{aligned}
D_{2}^{a}= & \mathcal{P}_{\delta b} T_{\rho} E_{\beta \alpha}^{\rho \delta} T^{\alpha \beta\{a b\}}-\mathcal{P}_{\gamma b}\left\{V_{\beta}^{\gamma}, T_{\alpha}\right\} \hat{T}^{\alpha \beta} \varepsilon^{a b} \\
& -\frac{1}{2} \lambda_{\gamma}\left(\left\{H_{0}, U_{\beta \alpha}^{\gamma}\right\}+V_{\delta}^{\gamma} U_{\beta \alpha}^{\delta}-U_{\beta \delta}^{\gamma} V_{\alpha}^{\delta}+(-1)^{\varepsilon_{\alpha} \varepsilon_{\beta}} U_{\alpha \delta}^{\gamma} V_{\beta}^{\delta}\right. \\
& \left.-2\left\{V_{\beta}^{\gamma}, T_{\alpha}\right\}\right)(-1)^{\varepsilon_{\beta}} C^{\alpha a} \pi^{\beta} \\
B_{3}^{a b}= & -\mathcal{P}_{\delta c} T_{\rho} E_{\gamma \beta \alpha}^{\rho \delta} T^{\alpha \beta \gamma\{a b c\}}-\frac{1}{3} \mathcal{P}_{\rho c}\left(\left\{U_{\beta \alpha}^{\rho}, T_{\gamma}\right\}(-1)^{\varepsilon_{\gamma}\left(\varepsilon_{\alpha}+\varepsilon_{\beta}\right)}\right. \\
& \left.+\frac{1}{2} U_{\gamma \delta}^{\rho} U_{\beta \alpha}^{\delta}\right)(-1)^{\varepsilon_{\beta}+\varepsilon_{\gamma}} C^{\alpha\{a} \varepsilon^{b\}} \hat{T}^{\beta \gamma} \\
& -\lambda_{\rho}\left(\frac{1}{2}(-1)^{\varepsilon_{\alpha} \varepsilon_{\gamma}} U_{\gamma \delta}^{\rho} U_{\beta \alpha}^{\delta}+\frac{1}{2}(-1)^{\varepsilon_{\alpha} \varepsilon_{\beta}} U_{\alpha \delta}^{\rho} U_{\gamma \beta}^{\delta}\right. \\
& \left.-(-1)^{\varepsilon_{\alpha} \varepsilon_{\gamma}}\left\{U_{\gamma \beta}^{\rho}, T_{\alpha}\right\}\right)(-1)^{\varepsilon_{\alpha} \varepsilon_{\gamma}} T^{\alpha \beta\{a b\}} \pi^{\gamma},
\end{aligned}
$$

To solve Eqs. (2.11) with the right-hand side (2.35) it is necessary to take into account the structural relations (2.33) and the identity

$$
E_{\alpha \beta}^{\gamma \delta} \pi^{\beta} \pi^{\alpha} \equiv 0
$$


which follows from the properties of generalized antisymmetry (2.32) for the structural coeffitients $E_{\alpha \beta}^{\gamma \delta}$. In turn, solving Eqs. (2.12) with the right-hand side (2.36) involves making use of the relations (2.34) with allowance made for the obvious identity

$$
\left(E_{\alpha \beta \gamma}^{\delta \rho}(-1)^{\varepsilon_{\alpha} \varepsilon_{\gamma}}-(-1)^{\varepsilon_{\alpha} \varepsilon_{\beta}} E_{\beta \alpha \gamma}^{\delta \rho}(-1)^{\varepsilon_{\beta} \varepsilon_{\gamma}}\right) \pi^{\beta} \pi^{\alpha} \equiv 0 .
$$

It is noteworthy that the methods of solving the equations for $\mathcal{H}_{2}$ and $\Omega_{3}^{a}$ are quite similar to each other.

Now, turnig ourselves to solution of the equations for $\mathcal{H}_{2}$, note that the structures present in $D_{2}^{a}$

$$
\mathcal{P}_{\gamma b} T_{\delta} E_{\beta \alpha}^{\delta \gamma} T^{\alpha \beta\{a b\}}, \quad \mathcal{P}_{\gamma b}\left\{V_{\beta}^{\gamma}, T_{\alpha}\right\} \hat{T}^{\alpha \beta} \varepsilon^{a b}
$$

could be reproduced by applying the operators $W^{a}$ respectively to the functions

$$
\frac{1}{2} \mathcal{P}_{\gamma a} \mathcal{P}_{\delta b}(-1)^{\varepsilon_{\gamma}} E_{\beta \alpha}^{\delta \gamma} T^{\alpha \beta\{a b\}}, \quad \lambda_{\gamma}\left\{V_{\beta}^{\gamma}, T_{\alpha}\right\} \hat{T}^{\alpha \beta}
$$

namely

$$
\begin{aligned}
W^{a}\left(-\frac{1}{2} \mathcal{P}_{\gamma b} \mathcal{P}_{\delta c}(-1)^{\varepsilon_{\gamma}} E_{\beta \alpha}^{\delta \gamma} T^{\alpha \beta\{b c\}}\right)= & \mathcal{P}_{\gamma b} T_{\delta} E_{\beta \alpha}^{\delta \gamma} T^{\alpha \beta\{a b\}} \\
& +\mathcal{P}_{\gamma b} \mathcal{P}_{\delta c}(-1)^{\varepsilon_{\gamma}} E_{\beta \alpha}^{\delta \gamma}(-1)^{\varepsilon_{\beta}} C^{\alpha c} \pi^{\beta} \varepsilon^{a b}, \\
W^{a}\left(\lambda_{\gamma}\left\{V_{\beta}^{\gamma}, T_{\alpha}\right\} \hat{T}^{\alpha \beta}\right)= & \mathcal{P}_{\gamma b}\left\{V_{\beta}^{\gamma}, T_{\alpha}\right\} \hat{T}^{\alpha \beta} \varepsilon^{a b} \\
& -\frac{1}{2} \lambda_{\gamma}\left(\left\{V_{\beta}^{\gamma}, T_{\alpha}\right\}+(-1)^{\varepsilon_{\alpha} \varepsilon_{\beta}}\left\{V_{\alpha}^{\gamma}, T_{\beta}\right\}\right)(-1)^{\varepsilon_{\beta}} C^{\alpha a} \pi^{\beta} .
\end{aligned}
$$

Consider the function $Y_{1}$

$$
Y_{1}=-\frac{1}{2} \mathcal{P}_{\gamma a} \mathcal{P}_{\delta b}(-1)^{\varepsilon_{\gamma}} E_{\beta \alpha}^{\delta \gamma} T^{\alpha \beta\{a b\}}-\lambda_{\gamma}\left\{V_{\beta}^{\gamma}, T_{\alpha}\right\} \hat{T}^{\alpha \beta},
$$

then from comparison of $D_{2}^{a}$ with $W^{a} Y_{1}$ we find

$$
\begin{aligned}
W^{a} Y_{1}-D_{2}^{a}= & \mathcal{P}_{\gamma b} \mathcal{P}_{\delta c}(-1)^{\varepsilon_{\gamma}} E_{\beta \alpha}^{\delta \gamma}(-1)^{\varepsilon_{\beta}} C^{\alpha c} \pi^{\beta} \varepsilon^{a b} \\
& +\lambda_{\gamma} T_{\delta} E_{\beta \alpha}^{\delta \gamma}(-1)^{\varepsilon_{\beta}} C^{\alpha a} \pi^{\beta}
\end{aligned}
$$


It is clear from analysis of summands in the right-hand side (2.37) that the structures present there could be reproduced by applying the operators $W^{a}$ to the function $Y_{2}$ of the form

$$
Y_{2}=-\lambda_{\gamma} \mathcal{P}_{\delta a}(-1)^{\varepsilon_{\gamma}} E_{\beta \alpha}^{\delta \gamma}(-1)^{\varepsilon_{\beta}} C^{\alpha a} \pi^{\beta}
$$

Indeed,

$$
W^{a} Y_{2}=-\mathcal{P}_{\gamma b} \mathcal{P}_{\delta c}(-1)^{\varepsilon_{\gamma}} E_{\beta \alpha}^{\delta \gamma}(-1)^{\varepsilon_{\beta}} C^{\alpha c} \pi^{\beta} \varepsilon^{a b}-\lambda_{\gamma} T_{\delta} E_{\beta \alpha}^{\delta \gamma}(-1)^{\varepsilon_{\beta}} C^{\alpha a} \pi^{\beta}
$$

and we find that

$$
W^{a}\left(Y_{1}+Y_{2}\right)-D_{2}^{a}=0
$$

Consequently, the solution to Eqs. (2.11) with the right-hand side (2.35) has the form

$$
\begin{aligned}
\mathcal{H}_{2}=Y_{1}+Y_{2}= & -\frac{1}{2} \mathcal{P}_{\gamma a} \mathcal{P}_{\delta b}(-1)^{\varepsilon_{\gamma}} E_{\beta \alpha}^{\delta \gamma} T^{\alpha \beta\{a b\}} \\
& -\lambda_{\gamma} \mathcal{P}_{\delta a}(-1)^{\varepsilon_{\gamma}} E_{\beta \alpha}^{\delta \gamma}(-1)^{\varepsilon_{\beta}} C^{\alpha a} \pi^{\beta}-\lambda_{\gamma}\left\{V_{\beta}^{\gamma}, T_{\alpha}\right\} \hat{T}^{\alpha \beta} .
\end{aligned}
$$

Omitting details of calculation of the functions $\Omega_{3}^{a}$, we only give here their resultant form

$$
\begin{aligned}
\Omega_{3}^{a}= & -\frac{1}{4} \mathcal{P}_{\delta b} \mathcal{P}_{\rho c}(-1)^{\varepsilon_{\delta}} E_{\gamma \beta \alpha}^{\rho \delta} T^{\alpha \beta \gamma\{a b c\}}+\frac{1}{2} \lambda_{\delta} \mathcal{P}_{\rho b}(-1)^{\varepsilon_{\delta}} E_{\gamma \beta \alpha}^{\rho \delta}(-1)^{\varepsilon_{\alpha} \varepsilon_{\gamma}} T^{\alpha \beta\{a b\}} \pi^{\gamma} \\
& +\frac{1}{3} \lambda_{\rho}\left(\frac{1}{2} U_{\gamma \delta}^{\rho} U_{\beta \alpha}^{\delta}+(-1)^{\varepsilon_{\gamma}\left(\varepsilon_{\alpha}+\varepsilon_{\beta}\right)}\left\{U_{\beta \alpha}^{\rho}, T_{\gamma}\right\}\right)(-1)^{\varepsilon_{\beta}+\varepsilon_{\gamma}} C^{\alpha a} \hat{T}^{\beta \gamma} .
\end{aligned}
$$

The explicit form of the functions $\mathcal{H}_{[2]}(2.23),(2.38)$ and $\Omega_{[3]}^{a}(2.10)$, (2.25), (2.39) obtained above enables us to consider the solution of Eqs. (2.11) for $\mathcal{H}_{3}$. To this end, it is necessary, as before, to turn ourselves to subsequent gauge algebra structural relations, being of the form

$$
\begin{aligned}
T_{\delta} E_{\alpha \beta \gamma}^{\delta \rho \sigma}= & -\frac{1}{12}\left\{H_{0}, E_{\alpha \beta \gamma}^{\rho \sigma}\right\}-\frac{1}{12}\left(V_{\delta}^{\rho} E_{\alpha \beta \gamma}^{\delta \sigma}-(-1)^{\varepsilon_{\rho} \varepsilon_{\sigma}} V_{\delta}^{\sigma} E_{\alpha \beta \gamma}^{\delta \rho}\right) \\
& +\frac{1}{4} E_{\alpha \beta \delta}^{\rho \sigma} V_{\gamma}^{\delta}(-1)^{\varepsilon_{\alpha}\left(\varepsilon_{\gamma}+\varepsilon_{\delta}\right)}+\frac{1}{6}(-1)^{\varepsilon_{\alpha} \varepsilon_{\gamma}}\left(E_{\alpha \delta}^{\rho \sigma} U_{\beta \gamma}^{\delta}-\left\{E_{\alpha \beta}^{\rho \sigma}, T_{\gamma}\right\}\right) \\
& +\frac{1}{12}(-1)^{\varepsilon_{\alpha} \varepsilon_{\gamma}}\left(\left\{V_{\alpha}^{\rho}, U_{\beta \gamma}^{\sigma}\right\}(-1)^{\varepsilon_{\alpha} \varepsilon_{\sigma}}-(-1)^{\varepsilon_{\rho} \varepsilon_{\sigma}}\left\{V_{\alpha}^{\sigma}, U_{\beta \gamma}^{\rho}\right\}(-1)^{\varepsilon_{\alpha} \varepsilon_{\rho}}\right) \\
& +\frac{1}{6}(-1)^{\varepsilon_{\alpha} \varepsilon_{\gamma}}\left(U_{\alpha \delta}^{\rho} E_{\beta \gamma}^{\delta \sigma}(-1)^{\varepsilon_{\alpha} \varepsilon_{\sigma}}-(-1)^{\varepsilon_{\rho} \varepsilon_{\sigma}} U_{\alpha \delta}^{\sigma} E_{\beta \gamma}^{\delta \rho}(-1)^{\varepsilon_{\alpha} \varepsilon_{\rho}}\right) \\
& + \text { cycl.perm. }(\alpha \beta \gamma),
\end{aligned}
$$


where the structural coeffitients $E_{\alpha \beta \gamma}^{\delta \rho \sigma}$ possess the properties

$$
E_{\alpha \beta \gamma}^{\delta \rho \sigma}=-(-1)^{\varepsilon_{\delta} \varepsilon_{\rho}} E_{\alpha \beta \gamma}^{\rho \delta \sigma}=-(-1)^{\varepsilon_{\rho} \varepsilon_{\sigma}} E_{\alpha \beta \gamma}^{\delta \sigma \rho}, \quad E_{\alpha \beta \gamma}^{\delta \rho \sigma}=E_{\gamma \alpha \beta}^{\delta \rho \sigma} .
$$

Validity of (2.40), (2.41) follows from the structural relations (2.33), (2.34) with allowance made for the Jacobi identities

$$
\begin{gathered}
\left\{\left\{T_{\alpha}, T_{\beta}\right\}, V_{\gamma}^{\delta}\right\}(-1)^{\varepsilon_{\alpha}\left(\varepsilon_{\gamma}+\varepsilon_{\delta}\right)}+\left\{\left\{T_{\beta}, V_{\gamma}^{\delta}\right\}, T_{\alpha}\right\}(-1)^{\varepsilon_{\alpha} \varepsilon_{\beta}} \\
+\left\{\left\{V_{\gamma}^{\delta}, T_{\alpha}\right\}, T_{\beta}\right\}(-1)^{\varepsilon_{\beta}\left(\varepsilon_{\gamma}+\varepsilon_{\delta}\right)} \equiv 0, \\
\left\{\left\{T_{\alpha}, U_{\beta \gamma}^{\delta}\right\}, H_{0}\right\}+\left\{\left\{H_{0}, T_{\alpha}\right\}, U_{\beta \gamma}^{\delta}\right\}+\left\{\left\{U_{\beta \gamma}^{\delta}, H_{0}\right\}, T_{\alpha}\right\}(-1)^{\varepsilon_{\alpha}\left(\varepsilon_{\beta}+\varepsilon_{\gamma}+\varepsilon_{\delta}\right)} \equiv 0,
\end{gathered}
$$

which could be presented respectively in the form

$$
\begin{aligned}
T_{\delta}\left\{V_{\alpha}^{\rho}, U_{\beta \gamma}^{\delta}\right\}(-1)^{\varepsilon_{\delta}\left(\varepsilon_{\alpha}+\varepsilon_{\rho}\right)}= & \left(\left\{\left\{V_{\alpha}^{\rho}, T_{\beta}\right\}, T_{\gamma}\right\}-(-1)^{\varepsilon_{\beta} \varepsilon_{\gamma}}\left\{\left\{V_{\alpha}^{\rho}, T_{\gamma}\right\}, T_{\beta}\right\}\right) \\
& -\left\{V_{\alpha}^{\rho}, T_{\delta}\right\} U_{\beta \gamma}^{\delta},
\end{aligned}
$$

$$
\begin{aligned}
T_{\delta}\left\{V_{\alpha}^{\delta}, U_{\beta \gamma}^{\rho}\right\}(-1)^{\varepsilon_{\alpha}\left(\varepsilon_{\beta}+\varepsilon_{\gamma}+\varepsilon_{\rho}\right)}= & \left\{U_{\beta \gamma}^{\rho}, T_{\delta}\right\} V_{\alpha}^{\delta}+\left\{\left\{U_{\beta \gamma}^{\rho}, T_{\alpha}\right\}, H_{0}\right\} \\
& +\left\{\left\{H_{0}, U_{\beta \gamma}^{\rho}\right\}, T_{\alpha}\right\}
\end{aligned}
$$

Then, taking into account the definition (2.27) and the properties (2.29), we obtain the functions $D_{3}^{a}$ in the form

$$
\begin{aligned}
D_{3}^{a}= & \mathcal{P}_{\delta b} \mathcal{P}_{\rho c}(-1)^{\varepsilon_{\delta}} T_{\sigma} E_{\gamma \beta \alpha}^{\sigma \rho \delta} T^{\alpha \beta \gamma\{a b c\}}-\frac{1}{3} \mathcal{P}_{\rho b} \mathcal{P}_{\sigma c}(-1)^{\varepsilon_{\rho}}\left[2 U_{\gamma \delta}^{\sigma} E_{\beta \alpha}^{\delta \rho}(-1)^{\varepsilon_{\gamma} \varepsilon_{\rho}}\right. \\
& +U_{\gamma \delta}^{\rho} E_{\beta \alpha}^{\delta \sigma}(-1)^{\varepsilon_{\sigma}\left(\varepsilon_{\gamma}+\varepsilon_{\rho}\right)}-E_{\gamma \delta}^{\sigma \rho} U_{\beta \alpha}^{\delta}-2\left\{E_{\gamma \alpha}^{\sigma \rho}, T_{\beta}\right\}(-1)^{\varepsilon_{\alpha} \varepsilon_{\beta}} \\
& \left.+\left\{V_{\gamma}^{\sigma}, U_{\beta \alpha}^{\rho}\right\}(-1)^{\varepsilon_{\gamma} \varepsilon_{\rho}}+2\left\{V_{\gamma}^{\rho}, U_{\beta \alpha}^{\sigma}\right\}(-1)^{\varepsilon_{\sigma}\left(\varepsilon_{\gamma}+\varepsilon_{\rho}\right)}\right](-1)^{\varepsilon_{\beta}+\varepsilon_{\gamma}} \varepsilon^{a b} C^{\alpha c} \hat{T}^{\beta \gamma} \\
& +\lambda_{\rho} \mathcal{P}_{\sigma b}(-1)^{\varepsilon_{\rho}}\left[\frac{1}{2}\left(\left\{H_{0}, E_{\gamma \beta \alpha}^{\sigma \rho}\right\}+V_{\delta}^{\sigma} E_{\gamma \beta \alpha}^{\delta \rho}-(-1)^{\varepsilon_{\rho} \varepsilon_{\sigma}} V_{\delta}^{\rho} E_{\gamma \beta \alpha}^{\delta \sigma}\right)(-1)^{\varepsilon_{\alpha} \varepsilon_{\gamma}}\right. \\
& -\frac{1}{2}\left(E_{\gamma \beta \delta}^{\sigma \rho} V_{\alpha}^{\delta}(-1)^{\varepsilon_{\gamma}\left(\varepsilon_{\alpha}+\varepsilon_{\delta}\right)}+\text { cycl.perm }(\alpha \beta \gamma)\right)(-1)^{\varepsilon_{\alpha} \varepsilon_{\gamma}} \\
& +\frac{1}{2}\left\{V_{\gamma}^{\rho}, U_{\beta \alpha}^{\sigma}\right\}(-1)^{\varepsilon_{\sigma}\left(\varepsilon_{\gamma}+\varepsilon_{\rho}\right)}+\frac{1}{2}\left\{V_{\beta}^{\sigma}, U_{\gamma \alpha}^{\rho}\right\}(-1)^{\varepsilon_{\beta}\left(\varepsilon_{\gamma}+\varepsilon_{\rho}\right)}+\left\{E_{\gamma \beta}^{\sigma \rho}, T_{\alpha}\right\} \\
& -\frac{1}{2}\left(E_{\gamma \delta}^{\sigma \rho} U_{\beta \alpha}^{\delta}-(-1)^{\varepsilon_{\beta} \varepsilon_{\gamma}} E_{\beta \delta}^{\sigma \rho} U_{\gamma \alpha}^{\delta}\right)+\frac{1}{2}\left(U_{\gamma \delta}^{\rho} E_{\beta \alpha}^{\delta \sigma}(-1)^{\varepsilon_{\sigma}\left(\varepsilon_{\gamma}+\varepsilon_{\rho}\right)}\right.
\end{aligned}
$$




$$
\begin{aligned}
& \left.\left.-(-1)^{\varepsilon_{\gamma} \varepsilon_{\beta}} U_{\beta \delta}^{\rho} E_{\gamma \alpha}^{\delta \sigma}(-1)^{\varepsilon_{\sigma}\left(\varepsilon_{\beta}+\varepsilon_{\rho}\right)}\right)+U_{\beta \delta}^{\sigma} E_{\gamma \alpha}^{\delta \rho}(-1)^{\varepsilon_{\beta}\left(\varepsilon_{\gamma}+\varepsilon_{\rho}\right)}\right] T^{\alpha \beta\{a b\}} \pi^{\gamma} \\
& +\lambda_{\rho} \mathcal{P}_{\sigma b}(-1)^{\varepsilon_{\rho}}\left[\left\{E_{\gamma \beta}^{\sigma \rho}, T_{\alpha}\right\}+\frac{1}{2}\left\{V_{\beta}^{\sigma}, U_{\gamma \alpha}^{\rho}\right\}(-1)^{\varepsilon_{\beta}\left(\varepsilon_{\rho}+\varepsilon_{\gamma}\right)}\right. \\
& \left.+\frac{1}{2} U_{\beta \delta}^{\rho} E_{\gamma \alpha}^{\delta \sigma}(-1)^{\varepsilon_{\rho} \varepsilon_{\sigma}+\varepsilon_{\beta}\left(\varepsilon_{\gamma}+\varepsilon_{\sigma}\right)}+\frac{1}{2}(-1)^{\varepsilon_{\beta} \varepsilon_{\gamma}} E_{\beta \delta}^{\sigma \rho} U_{\gamma \alpha}^{\delta}\right] \varepsilon^{a b} \hat{T}^{\alpha \beta} \pi^{\gamma} \\
& -\lambda_{\rho}\left[\left\{\left\{V_{\gamma}^{\rho}, T_{\beta}\right\}, T_{\alpha}\right\}-\frac{1}{2}\left(\left\{V_{\gamma}^{\rho}, T_{\delta}\right\}+(-1)^{\varepsilon_{\gamma} \varepsilon_{\delta}}\left\{V_{\delta}^{\rho}, T_{\gamma}\right\}\right) U_{\beta \alpha}^{\delta}\right. \\
& -\frac{1}{2} U_{\alpha \delta}^{\rho}\left\{V_{\gamma}^{\delta}, T_{\beta}\right\}(-1)^{\varepsilon_{\alpha}\left(\varepsilon_{\beta}+\varepsilon_{\gamma}\right)}-\frac{1}{3}\left\{H_{0},\left\{U_{\beta \alpha}^{\rho}, T_{\gamma}\right\}\right\}(-1)^{\varepsilon_{\gamma}\left(\varepsilon_{\alpha}+\varepsilon_{\beta}\right)} \\
& -\frac{1}{6}\left\{H_{0}, U_{\gamma \delta}^{\rho} U_{\beta \alpha}^{\delta},\right\}-\frac{1}{3} V_{\delta}^{\rho}\left(\left\{U_{\beta \alpha}^{\delta}, T_{\gamma}\right\}(-1)^{\varepsilon_{\gamma}\left(\varepsilon_{\alpha}+\varepsilon_{\beta}\right)}+\frac{1}{2} U_{\gamma \sigma}^{\delta} U_{\beta \alpha}^{\sigma}\right) \\
& +\frac{1}{3}\left(\left\{U_{\beta \delta}^{\rho}, T_{\gamma}\right\}(-1)^{\varepsilon_{\gamma}\left(\varepsilon_{\beta}+\varepsilon_{\delta}\right)}+\frac{1}{2} U_{\gamma \sigma}^{\rho} U_{\beta \delta}^{\sigma}\right) V_{\alpha}^{\delta} \\
& +\frac{1}{3}\left(\left\{U_{\gamma \alpha}^{\rho}, T_{\delta}\right\}(-1)^{\varepsilon_{\alpha} \varepsilon_{\delta}}+(-1)^{\varepsilon_{\gamma} \varepsilon_{\delta}}\left\{U_{\delta \alpha}^{\rho}, T_{\gamma}\right\}(-1)^{\varepsilon_{\alpha} \varepsilon_{\gamma}}\right. \\
& \left.\left.+\frac{1}{2} U_{\gamma \sigma}^{\rho} U_{\delta \alpha}^{\sigma}+\frac{1}{2}(-1)^{\varepsilon_{\gamma} \varepsilon_{\delta}} U_{\delta \sigma}^{\rho} U_{\gamma \alpha}^{\sigma}\right) V_{\beta}^{\delta}(-1)^{\varepsilon_{\alpha}\left(\varepsilon_{\beta}+\varepsilon_{\delta}\right)}\right] C^{\alpha a} \hat{T}^{\beta \gamma} \\
& -\frac{1}{2} \lambda_{\rho} \lambda_{\sigma}\left[\left\{V_{\gamma}^{\sigma}, U_{\beta \alpha}^{\rho}\right\}+U_{\gamma \delta}^{\sigma} E_{\beta \alpha}^{\delta \rho}\right](-1)^{\varepsilon_{\beta}+\varepsilon_{\gamma}\left(\varepsilon_{\rho}+1\right)} C^{\alpha a} \pi^{\beta} \pi^{\gamma} .
\end{aligned}
$$

Eqs. (2.11) for $\mathcal{H}_{3}$ are solved by the method given above. It implies consideration of summands present in $D_{3}^{a}$, taking into account the gauge algebra structural relations $(2.33),(2.34),(2.40),(2.42)$, and the properties of structural coeffitients $(2.2),(2.32),(2.41)$ with allowance made for the following identity

$$
\varepsilon_{a b}\left(C^{\alpha a} C^{\beta b} C^{\gamma c}+\text { cycl.perm. }(a b c)\right) \equiv 0 .
$$

Omitting details of calculation, we give here the resultant form of $\mathcal{H}_{3}$

$$
\begin{aligned}
\mathcal{H}_{3}= & \frac{1}{3} \mathcal{P}_{\delta a} \mathcal{P}_{\rho b} \mathcal{P}_{\sigma c}(-1)^{\varepsilon_{\rho}} E_{\gamma \beta \alpha}^{\sigma \rho \delta} T^{\alpha \beta \gamma\{a b c\}} \\
& +\lambda_{\delta} \mathcal{P}_{\rho a} \mathcal{P}_{\sigma b}(-1)^{\varepsilon_{\rho}} E_{\gamma \beta \alpha}^{\sigma \rho \delta}(-1)^{\varepsilon_{\alpha} \varepsilon_{\gamma}} T^{\alpha \beta\{a b\}} \pi^{\gamma} \\
& +\frac{1}{3} \lambda_{\rho} \mathcal{P}_{\sigma a}(-1)^{\varepsilon_{\rho}}\left[E_{\gamma \delta}^{\sigma \rho} U_{\beta \alpha}^{\delta}+2\left(\left\{E_{\gamma \alpha}^{\sigma \rho}, T_{\beta}\right\}(-1)^{\varepsilon_{\alpha} \varepsilon_{\beta}}\right.\right. \\
& \left.-U_{\gamma \delta}^{\sigma} E_{\beta \alpha}^{\delta \rho}(-1)^{\varepsilon_{\gamma} \varepsilon_{\rho}}\right)-U_{\gamma \delta}^{\rho} E_{\beta \alpha}^{\delta \sigma}(-1)^{\varepsilon_{\sigma}\left(\varepsilon_{\gamma}+\varepsilon_{\rho}\right)} \\
& \left.-\left\{V_{\gamma}^{\sigma}, U_{\beta \alpha}^{\rho}\right\}(-1)^{\varepsilon_{\gamma} \varepsilon_{\rho}}-2\left\{V_{\gamma}^{\rho}, U_{\beta \alpha}^{\sigma}\right\}(-1)^{\varepsilon_{\sigma}\left(\varepsilon_{\gamma}+\varepsilon_{\rho}\right)}\right](-1)^{\varepsilon_{\beta}+\varepsilon_{\gamma}} C^{\alpha a} \hat{T}^{\beta \gamma} \\
& +\lambda_{\rho} \lambda_{\sigma}\left[\left\{V_{\beta}^{\sigma}, U_{\gamma \alpha}^{\rho}\right\}+U_{\beta \delta}^{\sigma} E_{\gamma \alpha}^{\delta \rho}\right](-1)^{\varepsilon_{\beta}\left(\varepsilon_{\gamma}+\varepsilon_{\rho}\right)} \hat{T}^{\alpha \beta} \pi^{\gamma} .
\end{aligned}
$$

In view of (2.43) and the results obtained above (2.23), (2.25), (2.38), (2.39), 
we conclude that in the framework of the $\mathrm{Sp}(2)$-covariant version of generalized canonical quantization, as well as in its standard formulation, solutions to the generating equations are completely defined by the gauge algebra structural coeffitients. The boson function

$$
\begin{aligned}
\mathcal{H}\left(P_{A}, Q^{A}\right)= & H_{0}\left(p_{\imath}, q^{\imath}\right)+\mathcal{H}_{1}\left(P_{A}, Q^{A}\right)+\mathcal{H}_{2}\left(P_{A}, Q^{A}\right) \\
& +\mathcal{H}_{3}\left(P_{A}, Q^{A}\right)+O\left(C^{4-n} \pi^{n}\right), \quad 0 \leq n \leq 4
\end{aligned}
$$

and the fermion functions

$$
\begin{aligned}
\Omega^{a}\left(P_{A}, Q^{A}\right)= & \Omega_{1}^{a}\left(P_{A}, Q^{A}\right)+\Omega_{2}^{a}\left(P_{A}, Q^{A}\right)+\Omega_{3}^{a}\left(P_{A}, Q^{A}\right) \\
& +O\left(C^{4-n} \pi^{n}\right), \quad 0 \leq n \leq 4
\end{aligned}
$$

satisfy the gauge algebra generating equations (2.4) up to the $3 \mathrm{~d}$ order inclusively. As regards the description of arbitrariness in the solutions to the generating equations, note that this question has been thoroughly studied in Ref. 18.

Concluding, let us consider the special case of dynamical systems with constant structural coeffitients, such that

$$
\left\{V_{\alpha}^{\gamma}, T_{\beta}\right\}=\left\{U_{\alpha \beta}^{\delta}, T_{\gamma}\right\}=\left\{U_{\alpha \beta}^{\delta}, V_{\gamma}^{\rho}\right\}=0
$$

given this, we assume that

$$
E_{\alpha \beta}^{\gamma \delta}=E_{\alpha \beta \gamma}^{\delta \rho}=E_{\alpha \beta \gamma}^{\delta \rho \sigma}=0
$$

Then the functions $\mathcal{H}$ and $\Omega^{a}$ take on the form

$$
\begin{gathered}
\mathcal{H}=\mathcal{H}_{[1]}=H_{0}+\mathcal{P}_{\beta a} V_{\alpha}^{\beta} C^{\alpha a}+\lambda_{\beta} V_{\alpha}^{\beta} \pi^{\alpha} \\
\Omega^{a}=\Omega_{[3]}^{a}=T_{\alpha} C^{\alpha a}+\varepsilon^{a b} \mathcal{P}_{\alpha b} \pi^{\alpha}+\frac{1}{2} \mathcal{P}_{\gamma b} U_{\beta \alpha}^{\gamma} T^{\alpha \beta\{a b\}}-\frac{1}{2} \lambda_{\gamma} U_{\beta \alpha}^{\gamma}(-1)^{\varepsilon_{\beta}} C^{\alpha a} \pi^{\beta} \\
+\frac{1}{6} \lambda_{\rho} U_{\gamma \delta}^{\rho} U_{\beta \alpha}^{\delta}(-1)^{\varepsilon_{\beta}+\varepsilon_{\gamma}} C^{\alpha a} \hat{T}^{\beta \gamma} .
\end{gathered}
$$


In a particular case of dynamical systems of rank 1 with a closed algebra where all the constraints $T_{\alpha}$ are boson functions (i. e. $\varepsilon_{\alpha}=0$ ), this result coincide with the exact solution to the generating equations for the functions $\mathcal{H}$ and $\Omega^{a}$, obtained in Ref. 12 .

\section{The Solution of Equations for $\mathrm{S}$}

Now, turning to the Lagrangian version of the $\operatorname{Sp}(2)$-covariant quantization method, consider the classical theory of fields $A^{\imath}, \varepsilon\left(A^{\imath}\right) \equiv \varepsilon_{i}$, described by the action $\mathcal{L}=\mathcal{L}(A)$ invariant under the gauge transformations $\delta A^{\imath}=\mathcal{R}_{\alpha}^{\imath}(A) \xi^{\alpha}$

$$
\mathcal{L}_{, i}(A) \mathcal{R}_{\alpha}^{\imath}(A)=0
$$

where $\mathcal{R}_{\alpha}^{\imath}(A)$ are generators of gauge transformations $\varepsilon\left(\mathcal{R}_{\alpha}^{\imath}(A)\right)=\varepsilon_{\imath}+\varepsilon_{\alpha}$ and $\xi^{\alpha}$ are arbitrary functions $\varepsilon\left(\xi^{\alpha}\right) \equiv \varepsilon_{\alpha}$. Let us now introduce, in accordance with Refs.15, 17, the total configuration space $\Phi^{A}\left(\varepsilon\left(\Phi^{A}\right) \equiv \varepsilon^{A}\right)$ whose manifest structure is determined by the fact whether the set of generators is lineary independent (irreducible theories) or lineary dependent (reducible theories). In what follows we shall restrict ourselves to consideration of irreducible theories. The total configuration space of the theories in question has the following structure

$$
\begin{aligned}
\Phi^{A} & =\left(A^{\imath}, B^{\alpha}, C^{\alpha a}\right), \\
\operatorname{ngh}\left(A^{\imath}\right) & =0, \operatorname{ngh}\left(C^{\alpha a}\right)=1, \operatorname{ngh}\left(B^{\alpha}\right)=2
\end{aligned}
$$

on account of extention of the initial configuration space $A^{\imath}$ by introducing additional fields $B^{\alpha}\left(\varepsilon\left(B^{\alpha}\right)=\varepsilon_{\alpha}\right)$ and $\operatorname{Sp}(2)$ doublets of ghost fields $C^{\alpha a}\left(\varepsilon\left(C^{\alpha a}\right)=\varepsilon_{\alpha}+1\right)$. We also introduce the sets of anifields $\Phi_{A a}^{*}$ and $\bar{\Phi}_{A}$

$$
\begin{aligned}
& \Phi_{A a}^{*}=\left(A_{\imath a}^{*}, B_{\alpha a}^{*}, C_{\alpha a b}^{*}\right), \bar{\Phi}_{A}=\left(\bar{A}_{\imath}, \bar{B}_{\alpha}, \bar{C}_{\alpha a}\right), \\
& \quad \varepsilon\left(\Phi_{A a}^{*}\right)=\varepsilon_{A}+1, \varepsilon\left(\bar{\Phi}_{A}\right)=\varepsilon_{A}, \\
& \operatorname{ngh}\left(\Phi_{A a}^{*}\right)=-1-\operatorname{ngh}\left(\Phi^{A}\right), \operatorname{ngh}\left(\bar{\Phi}_{A}\right)=-2-\operatorname{ngh}\left(\Phi^{A}\right) .
\end{aligned}
$$


The antifields $\Phi_{A a}^{*}$ play the role of sources of BRST and anti-BRST transformations, whereas the antifields $\bar{\Phi}_{A}$ are the sources of mixed BRST and anti-BRST transformations.

The basic object in the Lagrangian version of $\operatorname{Sp}(2)$-covariant quantization $^{15,17}$ is the boson functional $S$, which satisfies the following generating equations

$$
\frac{1}{2}(S, S)^{a}+V^{a} S=i \hbar \Delta^{\alpha} S
$$

with the boundary condition

$$
S_{\mid \Phi_{a}^{*}=\bar{\Phi}=\hbar=0}=\mathcal{L}(A) .
$$

In (3.3) we used designation $(,)^{a}$ for an extended antibracket ${ }^{15}$ introduced for two arbitrary functionals $F$ and $G$ by the rule

$$
(F, G)^{a}=\frac{\delta F}{\delta \Phi^{A}} \frac{\delta G}{\delta \Phi_{A a}^{*}}-\frac{\delta G}{\delta \Phi^{A}} \frac{\delta F}{\delta \Phi_{A a}^{*}}(-1)^{(\varepsilon(F)+1)(\varepsilon(G)+1)},
$$

$V^{a}$ and $\Delta^{a}$ are operators defined in the form

$$
V^{a}=\varepsilon^{a b} \Phi_{A b}^{*} \frac{\delta}{\delta \bar{\Phi}_{A}}, \quad \Delta^{a}=(-1)^{\varepsilon_{A}} \frac{\delta_{l}}{\delta \Phi^{A}} \frac{\delta}{\delta \Phi_{A a}^{*}},
$$

$\hbar$ is the Plank constant. The algebra of operators $V^{a}$ and $\Delta^{a}$ as well as the properties of the extended antibracket are studied in detail in Ref. 15, and we shall not discuss here these questions.

The generating functional of the Green's functions $Z(J)$ for the fields of the extended configuration space is representable in the form ${ }^{15}$

$$
\begin{aligned}
Z(J)= & \int d \Phi d \Phi_{a}^{*} d \bar{\Phi} d \lambda d \Pi^{a} \exp \left\{\frac { i } { \hbar } \left(S\left(\Phi, \Phi_{a}^{*}, \bar{\Phi}\right)+\Phi_{A a}^{*} \Pi^{A a}\right.\right. \\
& \left.\left.+\left(\bar{\Phi}_{A}-\frac{\delta F}{\delta \Phi^{A}}\right) \lambda^{A}-\frac{1}{2} \varepsilon_{a b} \Pi^{A a} \frac{\delta^{2} F}{\delta \Phi^{A} \delta \Phi^{B}} \Pi^{B b}+J_{A} \Phi^{A}\right)\right\}
\end{aligned}
$$

where $J_{A}$ are the usual sources to the fields $\Phi^{A}\left(\varepsilon\left(J_{A}\right)=\varepsilon_{A}\right), \Pi^{A a}$ and $\lambda^{A}$ are the sets of auxiliary fields $\left(\varepsilon\left(\Pi^{A a}\right)=\varepsilon_{A}+1, \varepsilon\left(\lambda^{A}\right)=\varepsilon_{A}\right), F=F(\Phi)$ is the boson gauge functional, $S\left(\Phi, \Phi_{a}^{*}, \bar{\Phi}\right)$ is a solution to Eqs.(3.3) with the boundary condition (3.4). 
An impotant property of the integrand in Eqs.(3.6) for $J_{A}=0$ is its invariance under the following transformations of global supersymmetry

$$
\begin{aligned}
\delta \Phi^{A}= & \Pi^{A a} \mu_{a}, \delta \Phi_{A a}^{*}=\mu_{a} \frac{\delta S}{\delta \Phi^{A}}, \delta \bar{\Phi}_{A}=\varepsilon^{a b} \mu_{a} \Phi_{A b}^{*}, \\
& \delta \Pi^{A a}=-\varepsilon^{a b} \lambda^{A} \mu_{b}, \delta \lambda^{A}=0
\end{aligned}
$$

where $\mu_{a}$ is a doublet of constant anticommuting Grassmann parameters. The transformations (3.7) realize the extended BRST transformations in the space of the variables $\Phi, \Phi_{a}^{*}, \bar{\Phi}, \Pi^{a}, \lambda$.

The symmetry of the vacuum functional $Z(0)$ under the transformations (3.7) permits establishing the independence of the $\mathrm{S}$ matrix on the choice of a gauge. Indeed, suppose $Z_{F} \equiv Z(0)$. We shall now change the gauge $F \rightarrow F+\Delta F$. In the functional integral for $Z_{F+\Delta F}$ we make the change of variables (3.7), choosing for the parameters $\mu_{a}$

$$
\mu_{a}=\frac{i}{2 \hbar} \varepsilon_{a b} \frac{\delta(\Delta F)}{\delta \Phi^{A}} \Pi^{A b}
$$

we find that $Z_{F+\Delta F}=Z_{F}$ and therefore the $\mathrm{S}$ matrix is gauge invariant. Next, the extended BRST symmetry permits deriving the Ward identities for the generating functional of the vertex functions (the effective action) $\Gamma=\Gamma\left(\Phi, \Phi_{a}^{*}, \bar{\Phi}\right)$

$$
\frac{1}{2}(\Gamma, \Gamma)^{a}+V^{a} \Gamma=0
$$

The identities for $\Gamma$ provide a basis for establishing the gauge invariant renormalizability of the general gauge theories in the framework of the $\operatorname{Sp}(2)$ - covariant quantization. ${ }^{17}$

In Refs. 15, 17 there is proved the existence theorem for solution to Eqs. (3.3) in the form of expansion in power series of $\hbar$

$$
S\left(\Phi, \Phi_{a}^{*}, \bar{\Phi}\right)=\sum_{n=0}^{\infty} \hbar^{n} S^{(n)}\left(\Phi, \Phi_{a}^{*}, \bar{\Phi}\right)
$$

where the functionals $S^{(n)}\left(\Phi, \Phi_{a}^{*}, \bar{\Phi}\right)$ are supposed to be regular with respect to all the variables. In particular, the tree (classical) approximation $S^{(0)}$ 
satisfies the following generating equations

$$
\frac{1}{2}\left(S^{(0)}, S^{(0)}\right)^{a}+V^{a} S^{(0)}=0 .
$$

In what follows we shall concentrate our attention upon Eqs. (3.8). One can seek solution to Eqs. (3.8) in the form of expansion in power series of ghost and auxiliary fields $C^{\alpha a}, B^{\alpha}$

$$
S^{(0)}\left(\Phi, \Phi_{a}^{*}, \bar{\Phi}\right)=\mathcal{L}(A)+\sum_{n=1}^{\infty} S_{n}\left(\Phi, \Phi_{a}^{*}, \bar{\Phi}\right),
$$

having required the new ghost number and the Grassmann parity to be conserved in every order of perturbation series

$$
\varepsilon\left(S_{n}\right)=\operatorname{ngh}\left(S_{n}\right)=0 .
$$

In (3.9) $S_{n}\left(\Phi, \Phi_{a}^{*}, \bar{\Phi}\right)$ are some $n$th order polinomials in the fields $C^{\alpha a}, B^{\alpha}$. The requirement of the new ghost number conservation leads to the fact that $S_{n}$ must be polinomials in the antifields $\Phi_{A a}^{*}, \bar{\Phi}_{A}$. In Ref. 15 it is shown that the first approximation $S_{1}$ could be chosen in the form

$$
S_{1}\left(\Phi, \Phi_{a}^{*}, \bar{\Phi}\right)=A_{\imath a}^{*} \mathcal{R}_{\alpha}^{\imath}(A) C^{\alpha a}+\bar{A}_{\imath} \mathcal{R}_{\alpha}^{\imath}(A) B^{\alpha}-\varepsilon^{a b} C_{\alpha a b}^{*} B^{\alpha}
$$

Then the higher approximations in (3.9) are sought from the equations

$$
W^{a} S_{n+1}=F_{n+1}^{a}, n \geq 1
$$

where the operators $W^{a}$ are defined in the form

$$
\begin{aligned}
W^{a}= & \mathcal{L}_{, \imath} \frac{\delta}{\delta A_{\imath a}^{*}}+A_{\imath b}^{*} \mathcal{R}_{\alpha}^{\imath} \frac{\delta}{\delta C_{\alpha a b}^{*}}+\left(\bar{A}_{\imath} \mathcal{R}_{\alpha}^{\imath}-\varepsilon^{b c} C_{\alpha b c}^{*}\right) \frac{\delta}{\delta B_{\alpha a}^{*}} \\
& +(-1)^{\varepsilon_{\alpha}} \varepsilon^{a b} B^{\alpha} \frac{\delta_{l}}{\delta C_{\alpha b}}+V^{a}
\end{aligned}
$$

and possess the properties

$$
W^{\{a} W^{b\}}=0 .
$$

The functionals $F_{n+1}^{a}$ are constucted from $S_{k}, k \leq n$ by the rule

$$
F_{n+1}^{a}=-\frac{1}{2}\left(S_{[n]}, S_{[n]}\right)_{n+1}^{a},
$$


where

$$
S_{[n]}=\mathcal{L}(A)+\sum_{k=1}^{n} S_{k}
$$

the symbol $(,)_{k}^{a}$ denotes the $k$ th order for the extended antibracket in power series of the fields $C^{\alpha a}$ and $B^{\alpha}$. The functionals $F_{n+1}^{a}$ satisfy the equations

$$
W^{\{a} F_{n+1}^{b\}}=0
$$

being the compability conditions for Eqs. (3.11).

Let us obtain the explicit solutions to Eqs. (3.11) when $n=1,2$ in the case of general gauge theories described by the action $\mathcal{L}(\mathcal{A})$ invariant under the gauge transformations (3.1). The algebra of generators $\mathcal{R}_{\alpha}^{\imath}(A)$ has the following general form

$$
\begin{gathered}
\mathcal{R}_{\alpha, \jmath}^{\imath}(A) \mathcal{R}_{\beta}^{\jmath}(A)-(-1)^{\varepsilon_{\alpha} \varepsilon_{\beta}} \mathcal{R}_{\beta, \jmath}^{\imath}(A) \mathcal{R}_{\alpha}^{\jmath}(A)= \\
-\mathcal{R}_{\gamma}^{\imath}(A) \mathcal{F}_{\alpha \beta}^{\gamma}(A)-\mathcal{L},_{\jmath}(A) M_{\alpha \beta}^{\imath \jmath}(A)
\end{gathered}
$$

where the structural coefficients $\mathcal{F}_{\alpha \beta}^{\gamma}(A)$ and $M_{\alpha \beta}^{\imath \jmath}(A)$ possess the properties of generalized antisymmetry

$$
\begin{gathered}
\mathcal{F}_{\alpha \beta}^{\gamma}(A)=-(-1)^{\varepsilon_{\alpha} \varepsilon_{\beta}} \mathcal{F}_{\beta \alpha}^{\gamma}(A), \\
M_{\alpha \beta}^{\imath \jmath}(A)=-(-1)^{\varepsilon_{\alpha} \varepsilon_{\beta}} M_{\beta \alpha}^{\imath \jmath}(A)=-(-1)^{\varepsilon_{2} \varepsilon_{j}} M_{\alpha \beta}^{\jmath \imath}(A) .
\end{gathered}
$$

Consider here the solution of Eqs. (3.11) for $S_{2}$. To do this we shall make use of the decomposition (2.18) of an $\mathrm{Sp}(2)$ tensor of rank two, constructed from $C^{\alpha a}$. Then, by virtue of the properties (2.19) and the algebra of generators (3.14), we find the functionals $F_{2}^{a}$ in the form

$$
\begin{aligned}
F_{2}^{a}= & -\frac{1}{2} A_{\imath d}^{*}\left(\mathcal{R}_{\gamma}^{\imath}(A) \mathcal{F}_{\alpha \beta}^{\gamma}(A)+\mathcal{L},_{\jmath}(A) M_{\alpha \beta}^{\imath \jmath}(A)\right) T^{\beta \alpha\{a d\}} \\
& +\frac{1}{2} \bar{A}_{\imath}\left(\mathcal{R}_{\gamma}^{\imath}(A) \mathcal{F}_{\alpha \beta}^{\gamma}(A)+\mathcal{L}_{, \jmath}(A) M_{\alpha \beta}^{\imath \jmath}(A)\right) C^{\beta a} B^{\alpha}(-1)^{\varepsilon_{\alpha}} \\
& +\frac{1}{2} \varepsilon^{a d} A_{\imath d}^{*} N_{\alpha \beta}^{\imath}(A) \hat{T}^{\beta \alpha}-\frac{1}{2} \bar{A}_{\imath} N_{\alpha \beta}^{\imath}(A) C^{\beta a} B^{\alpha}(-1)^{\varepsilon_{\alpha}}
\end{aligned}
$$


where

$$
\begin{gathered}
N_{\alpha \beta}^{\imath}(A)=\mathcal{R}_{\alpha, \jmath}^{\imath}(A) \mathcal{R}_{\beta}^{\jmath}(A)+(-1)^{\varepsilon_{\alpha} \varepsilon_{\beta}} \mathcal{R}_{\beta, \jmath}^{\imath}(A) \mathcal{R}_{\alpha}^{\jmath}(A), \\
N_{\alpha \beta}^{\imath}(A)=(-1)^{\varepsilon_{\alpha} \varepsilon_{\beta}} N_{\beta \alpha}^{\imath}(A)
\end{gathered}
$$

Solving Eqs. (3.11) for $S_{2}$ is as follows. Consider the operators $W^{a}$ to act upon the functional

$$
\begin{gathered}
\frac{1}{2} C_{\gamma b d}^{*} \mathcal{F}_{\alpha \beta}^{\gamma}(A) T^{\beta \alpha\{b d\}} \\
W^{a}\left(-\frac{1}{2} C_{\gamma b d}^{*} \mathcal{F}_{\alpha \beta}^{\gamma}(A) T^{\beta \alpha\{b d\}}\right)=-\frac{1}{2} A_{d d}^{*} \mathcal{R}_{\gamma}^{\imath}(A) \mathcal{F}_{\alpha \beta}^{\gamma}(A) T^{\beta \alpha\{a d\}} \\
-\frac{1}{2} \varepsilon^{a b}\left(C_{\gamma b d}^{*}+C_{\gamma d b}^{*}\right) \mathcal{F}_{\alpha \beta}^{\gamma}(A) B^{\beta} C^{\alpha d} .
\end{gathered}
$$

Hence we find that the first summand in (3.17) present in (3.16) as well. In a similar way, the structure of the form

$$
-\frac{1}{2} A_{\imath d}^{*} \mathcal{L},_{\jmath}(A) M_{\alpha \beta}^{\imath \jmath}(A) T^{\beta \alpha\{a d\}}
$$

is reprodused by applying the operators $W^{a}$ to the functional

$$
\frac{1}{4} A_{\imath b}^{*} A_{\jmath d}^{*}(-1)^{\varepsilon_{\imath}} M_{\alpha \beta}^{\imath \jmath}(A) T^{\beta \alpha\{b d\}},
$$

namely

$$
\begin{gathered}
W^{a}\left(\frac{1}{4} A_{\imath b}^{*} A_{\jmath d}^{*}(-1)^{\varepsilon_{\imath}} M_{\alpha \beta}^{\imath \jmath}(A) T^{\beta \alpha\{b d\}}\right)=-\frac{1}{2} A_{\imath d}^{*} \mathcal{L},{ }_{\jmath} M_{\alpha \beta}^{\imath \jmath}(A) T^{\beta \alpha\{a d\}} \\
+\frac{1}{2} \varepsilon^{a b} A_{\imath b}^{*} A_{\jmath d}^{*}(-1)^{\varepsilon_{\imath}} M_{\alpha \beta}^{\imath \jmath}(A) B^{\beta} C^{\alpha d} .
\end{gathered}
$$

Treatment of the terms

$$
\frac{1}{2} \bar{A}_{\imath} \mathcal{R}_{\gamma}^{\imath}(A) \mathcal{F}_{\alpha \beta}^{\gamma}(A) C^{\beta a} B^{\alpha}(-1)^{\varepsilon_{\alpha}}
$$

and

$$
\frac{1}{2} \bar{A}_{\imath} \mathcal{L},{ }_{j} M_{\alpha \beta}^{\imath \jmath}(A) C^{\beta a} B^{\alpha}(-1)^{\varepsilon_{\alpha}}
$$

present in $F_{2}^{a}$ consists in consideration of $W^{a}$ to act respectively upon

$$
\frac{1}{2} B_{\gamma b}^{*} \mathcal{F}_{\alpha \beta}^{\gamma}(A) C^{\beta b} B^{\alpha}(-1)^{\varepsilon_{\alpha}}
$$


and

$$
\frac{1}{2} \bar{A}_{\imath} A_{\jmath b}^{*}(-1)^{\varepsilon_{\imath}} M_{\alpha \beta}^{\imath \jmath}(A) C^{\beta b} B^{\alpha}(-1)^{\varepsilon_{\alpha}},
$$

namely

$$
\begin{gathered}
W^{a}\left(\frac{1}{2} B_{\gamma b}^{*} \mathcal{F}_{\alpha \beta}^{\gamma}(A) C^{\beta b} B^{\alpha}(-1)^{\varepsilon_{\alpha}}\right)=\frac{1}{2} \bar{A}_{\imath} \mathcal{R}_{\gamma}^{\imath}(A) \mathcal{F}_{\alpha \beta}^{\gamma}(A) C^{\beta a} B^{\alpha}(-1)^{\varepsilon_{\alpha}} \\
-\frac{1}{2} \varepsilon^{b d} C_{\gamma b d}^{*} \mathcal{F}_{\alpha \beta}^{\gamma}(A) C^{\beta a} B^{\alpha}(-1)^{\varepsilon_{\alpha}} \\
W^{a}\left(\frac{1}{2} \bar{A}_{\imath} A_{\jmath b}^{*}(-1)^{\varepsilon_{\imath}} M_{\alpha \beta}^{\imath \jmath}(A) C^{\beta b} B^{\alpha}(-1)^{\varepsilon_{\alpha}}\right)= \\
\frac{1}{2} \bar{A}_{\imath} \mathcal{L},_{\jmath}(A) M_{\alpha \beta}^{\imath \jmath}(A) C^{\beta a} B^{\alpha}(-1)^{\varepsilon_{\alpha}} \\
-\frac{1}{2} \varepsilon^{a b} A_{\imath b}^{*} A_{\jmath d}^{*}(-1)^{\varepsilon_{\imath}} M_{\alpha \beta}^{\imath \jmath}(A) B^{\beta} C^{\alpha d}
\end{gathered}
$$

Consider the functional of the form

$$
\begin{aligned}
X_{1}\left(\Phi^{A}, \Phi_{A a}^{*}, \bar{\Phi}_{A}\right)= & -\frac{1}{2} C_{\gamma b d}^{*} \mathcal{F}_{\alpha \beta}^{\gamma}(A) T^{\beta \alpha\{b d\}}+\frac{1}{2} B_{\gamma b}^{*} \mathcal{F}_{\alpha \beta}^{\gamma}(A) C^{\beta b} B^{\alpha}(-1)^{\varepsilon_{\alpha}} \\
& +\frac{1}{4} A_{\imath b}^{*} A_{\jmath d}^{*}(-1)^{\varepsilon_{\imath}} M_{\alpha \beta}^{\imath \jmath}(A) T^{\beta \alpha\{b d\}} \\
& +\frac{1}{2} \bar{A}_{\imath} A_{\jmath b}^{*}(-1)^{\varepsilon_{\imath}} M_{\alpha \beta}^{\imath \jmath}(A) C^{\beta b} B^{\alpha}(-1)^{\varepsilon_{\alpha}}
\end{aligned}
$$

then

$$
\begin{gathered}
W^{a} X_{1}=-\frac{1}{2} A_{\imath d}^{*} \mathcal{L},{ }_{\jmath} M_{\alpha \beta}^{\imath \jmath}(A) T^{\beta \alpha\{a d\}}+\frac{1}{2} \bar{A}_{\imath} \mathcal{L},{ }_{\jmath}(A) M_{\alpha \beta}^{\imath \jmath}(A) C^{\beta a} B^{\alpha}(-1)^{\varepsilon_{\alpha}} \\
-\frac{1}{2} A_{\imath d}^{*} \mathcal{R}_{\gamma}^{\imath}(A) \mathcal{F}_{\alpha \beta}^{\gamma}(A) T^{\beta \alpha\{a d\}}+\frac{1}{2} \bar{A}_{\imath} \mathcal{R}_{\gamma}^{\imath}(A) \mathcal{F}_{\alpha \beta}^{\gamma}(A) C^{\beta a} B^{\alpha}(-1)^{\varepsilon_{\alpha}} \\
-\frac{1}{2} \varepsilon^{a b}\left(C_{\gamma b d}^{*}+C_{\gamma d b}^{*}\right) \mathcal{F}_{\alpha \beta}^{\gamma}(A) B^{\beta} C^{\alpha d} \\
-\frac{1}{2} \varepsilon^{b d} C_{\gamma b d}^{*} \mathcal{F}_{\alpha \beta}^{\gamma}(A) C^{\beta a} B^{\alpha}(-1)^{\varepsilon_{\alpha}}
\end{gathered}
$$

The functional $X_{1}\left(\Phi^{A}, \Phi_{A a}^{*}, \bar{\Phi}_{A}\right)$ possesses the properties $\varepsilon\left(X_{1}\right)=\operatorname{ngh}\left(X_{1}\right)=$ 0 with the multipliers chosen in such a way that $W^{a} X_{1}$ should exhaust the summands in $F_{2}^{a}$ described above. In calculating $W^{a} X_{1}$ (3.22) one has to make use of the generalized antisymmetry properties (3.15) of the gauge algebra structural coeffitients $\mathcal{F}_{\alpha \beta}^{\gamma}(A)$ and $M_{\alpha \beta}^{\imath \jmath}(A)$ with allowance made for 
the properties (2.19) and for the definition of the tensors $T^{\beta \alpha\{a d\}}, \varepsilon^{a b} \hat{T}^{\beta \alpha}$ (2.18) and the properties (2.19). In particular,

$$
\mathcal{F}_{\alpha \beta}^{\gamma}(A) B^{\alpha} B^{\beta} \equiv M_{\alpha \beta}^{\imath \jmath}(A) B^{\alpha} B^{\beta} \equiv 0
$$

Comparison of $W^{a} X_{1}$ with $F_{2}^{a}$ implies

$$
\begin{aligned}
F_{2}^{a}-W^{a} X_{1}= & \frac{1}{2} \varepsilon^{a d} A_{\imath d}^{*} N_{\alpha \beta}^{\imath}(A) \hat{T}^{\beta \alpha}-\frac{1}{2} \bar{A}_{\imath} N_{\alpha \beta}^{\imath}(A) C^{\beta a} B^{\alpha}(-1)^{\varepsilon_{\alpha}} \\
& +\varepsilon^{a b} C_{\gamma b d}^{*} \mathcal{F}_{\alpha \beta}^{\gamma}(A) B^{\beta} C^{\alpha d}
\end{aligned}
$$

In establishing (3.24) we made use of the identity

$$
\begin{gathered}
\frac{1}{2} \varepsilon^{a b}\left(C_{\gamma b d}^{*}+C_{\gamma d b}^{*}\right) \mathcal{F}_{\alpha \beta}^{\gamma}(A) B^{\beta} C^{\alpha d}+\frac{1}{2} \varepsilon^{b d} C_{\gamma b d}^{*} \mathcal{F}_{\alpha \beta}^{\gamma}(A) B^{\beta} C^{\alpha a}= \\
\varepsilon^{a b} C_{\gamma b d}^{*} \mathcal{F}_{\alpha \beta}^{\gamma}(A) B^{\beta} C^{\alpha d},
\end{gathered}
$$

verified directly. Making an analysis similar to the one discussed above for each summand present in (3.24), consider $W^{a}$ to act upon the functional $X_{2}$ of the form

$$
X_{2}=\frac{1}{2} \bar{A}_{\imath} N_{\alpha \beta}^{\imath}(A) \hat{T}^{\beta \alpha}+\bar{C}_{\gamma d} \mathcal{F}_{\alpha \beta}^{\gamma}(A) B^{\beta} C^{\alpha d} .
$$

One readily finds that $F_{2}^{a}-W^{a}\left(X_{1}+X_{2}\right)=0$. Consequently, the solution to Eqs. (3.11) with $F_{2}^{a}(3.16)$ has the form

$$
\begin{aligned}
S_{2}=X_{1}+X_{2}= & -\frac{1}{2} C_{\gamma b d}^{*} \mathcal{F}_{\alpha \beta}^{\gamma}(A) T^{\beta \alpha\{b d\}}+\frac{1}{2} B_{\gamma b}^{*} \mathcal{F}_{\alpha \beta}^{\gamma}(A) C^{\beta b} B^{\alpha} \\
& +\frac{1}{4} A_{\imath b}^{*} A_{\jmath d}^{*}(-1)^{\varepsilon_{\imath}} M_{\alpha \beta}^{\imath \jmath}(A) T^{\beta \alpha\{b d\}} \\
& +\frac{1}{2} \bar{A}_{\imath} A_{\jmath b}^{*}(-1)^{\varepsilon_{\imath}} M_{\alpha \beta}^{\imath \jmath}(A) C^{\beta b} B^{\alpha}(-1)^{\varepsilon_{\alpha}} \\
& +\frac{1}{2} \bar{A}_{\imath} N_{\alpha \beta}^{\imath}(A) \hat{T}^{\beta \alpha}+\bar{C}_{\gamma d} \mathcal{F}_{\alpha \beta}^{\gamma}(A) B^{\beta} C^{\alpha d} .
\end{aligned}
$$

Consider now the solution of Eqs. (3.11) for $S_{3}$. It is convinient, as mentioned above, to seek the solution with allowance made for the decomposition (2.27) of an $\mathrm{Sp}(2)$ tensor of rank three constructed from $C^{\alpha a}$.

In solving Eqs. (3.11) for $S_{3}$ it is necessary to employ subsequent structural relations of the gauge algebra ${ }^{23}$, that is to say, the generalized Jacobi 
identity

$$
\mathcal{R}_{\gamma}^{\imath}(A) D_{\alpha \beta \delta}^{\gamma}(A)+\mathcal{L},{ }_{k} Z_{\alpha \beta \delta}^{\imath k}(A)=0
$$

where

$$
\begin{aligned}
D_{\beta \delta \sigma}^{\gamma}(A)= & (-1)^{\varepsilon_{\beta} \varepsilon_{\sigma}}\left(\mathcal{F}_{\beta \alpha}^{\gamma}(A) \mathcal{F}_{\delta \sigma}^{\alpha}(A)+\mathcal{F}_{\beta \delta, \imath}^{\gamma}(A) \mathcal{R}_{\sigma}^{\imath}(A)\right) \\
& + \text { cycl.perm. }(\beta \delta \sigma), \\
Z_{\beta \delta \sigma}^{\imath k}(A)= & (-1)^{\varepsilon_{\beta} \varepsilon_{\sigma}}\left(M_{\beta \alpha}^{\imath k}(A) \mathcal{F}_{\delta \sigma}^{\alpha}(A)+M_{\beta \delta, \jmath}^{\imath k}(A) \mathcal{R}_{\sigma}^{\jmath}(A)\right. \\
& \left.-(-1)^{\varepsilon_{\beta} \varepsilon_{\imath}} \mathcal{R}_{\beta, \jmath}^{k}(A) M_{\delta \sigma}^{\imath \jmath}(A)+(-1)^{\varepsilon_{k}\left(\varepsilon_{\imath}+\varepsilon_{\beta}\right)} \mathcal{R}_{\beta, \jmath}^{\imath}(A) M_{\delta \sigma}^{k \jmath}(A)\right) \\
& + \text { cycl.perm. }(\beta \delta \sigma),
\end{aligned}
$$

Given this

$$
\begin{aligned}
& D_{\beta \delta \sigma}^{\gamma}(A)=-(-1)^{\varepsilon_{\beta \delta \sigma}} D_{\delta \beta \sigma}^{\gamma}(A)=-(-1)^{\varepsilon_{\beta \delta \sigma}} D_{\beta \sigma \delta}^{\gamma}(A), \\
& Z_{\beta \delta \sigma}^{\imath k}(A)=-(-1)^{\varepsilon_{k} \varepsilon_{\imath}} Z_{\beta \delta \sigma}^{k \imath}(A)=-(-1)^{\varepsilon_{\beta \delta \sigma}} Z_{\delta \beta \sigma}^{\imath k}(A)= \\
& \quad-(-1)^{\varepsilon_{\beta \delta \sigma}} Z_{\beta \sigma \delta}^{\imath k}(A) .
\end{aligned}
$$

In Ref. 23 it is shown that Eq.(3.27) leads to the conclusion that there exist the gauge algebra structural coeffitients $Q_{\beta \delta \sigma}^{\mu \imath}(A)$ and $\mathcal{D}_{\beta \delta \sigma}^{\imath \jmath k}(A)$ such that

$$
\begin{gathered}
Z_{\beta \delta \sigma}^{\imath k}(A)+(-1)^{\varepsilon_{\mu} \varepsilon_{\imath}} \mathcal{R}_{\mu}^{k}(A) Q_{\beta \delta \sigma}^{\mu \imath}(A) \\
-(-1)^{\left(\varepsilon_{\mu}+\varepsilon_{\imath}\right) \varepsilon_{k}} \mathcal{R}_{\mu}^{\imath}(A) Q_{\beta \delta \sigma}^{\mu k}(A)=-{\mathcal{L},{ }_{\jmath}}(A) \mathcal{D}_{\beta \delta \sigma}^{\imath k \jmath}(A)
\end{gathered}
$$

with

$$
D_{\beta \delta \sigma}^{\gamma}(A)=\mathcal{L}_{, k}(A) Q_{\beta \delta \sigma}^{\gamma k}(A)
$$

Given this

$$
\begin{aligned}
& Q_{\beta \delta \sigma}^{\gamma k}(A)=-(-1)^{\varepsilon_{\beta \delta \sigma}} Q_{\delta \beta \sigma}^{\gamma k}(A)=-(-1)^{\varepsilon_{\beta \delta \sigma}} Q_{\beta \sigma \delta}^{\gamma k}(A), \\
& \mathcal{D}_{\beta \delta \sigma}^{\imath k J}(A)=-(-1)^{\varepsilon_{k} \varepsilon_{2}} \mathcal{D}_{\beta \delta \sigma}^{k \imath \jmath}(A)=-(-1)^{\varepsilon_{k} \varepsilon_{j}} \mathcal{D}_{\beta \delta \sigma}^{\imath \jmath}(A), \\
& \mathcal{D}_{\beta \delta \sigma}^{\imath k J}(A)=-(-1)^{\varepsilon_{\beta \delta \sigma}} \mathcal{D}_{\delta \beta \sigma}^{\imath k J}(A)=-(-1)^{\varepsilon_{\beta \delta \sigma}} \mathcal{D}_{\beta \sigma \delta}^{\imath k J}(A)
\end{aligned}
$$


Then, by virtue of the gauge algebra relations (3.27), we find from definition (2.27) and the properties (3.15), (2.19), (2.29), (3.28) the following representation for $F_{3}^{a}$

$$
\begin{aligned}
& F_{3}^{a}=\frac{1}{6} C_{\gamma b c}^{*} D_{\beta \delta \sigma}^{\gamma}(A) T^{\sigma \delta \beta\{a b c\}} \\
& +\frac{1}{6} \varepsilon^{c a}\left(C_{\gamma b c}^{*}+C_{\gamma c b}^{*}\right)\left[\mathcal{F}_{\delta \alpha}^{\gamma}(A) \mathcal{F}_{\beta \sigma}^{\alpha}(A)\right. \\
& \left.+2 \mathcal{F}_{\delta \beta, \imath}^{\gamma}(A) \mathcal{R}_{\sigma}^{\imath}(A)\right](-1)^{\varepsilon_{\beta} \varepsilon_{\delta}} \hat{T}^{\sigma \delta} C^{\beta b} \\
& -\frac{1}{12} A_{k b}^{*} A_{p c}^{*}(-1)^{\varepsilon_{k}} Z_{\beta \delta \sigma}^{k p}(A) T^{\sigma \delta \beta\{a b c\}} \\
& +\frac{1}{6} \varepsilon^{a b} A_{k b}^{*} A_{p c}^{*}(-1)^{\varepsilon_{k}}\left(M_{\beta \alpha}^{k p}(A) \mathcal{F}_{\delta \sigma}^{\alpha}(A)+2 M_{\beta \delta, \imath}^{k p}(A) \mathcal{R}_{\sigma}^{\imath}(A)\right. \\
& \left.-2 \mathcal{R}_{\beta, \imath}^{p}(A) M_{\delta \sigma}^{k l}(A)(-1)^{\varepsilon_{k} \varepsilon_{\beta}}\right)(-1)^{\varepsilon_{\delta} \varepsilon_{\beta}} \hat{T}^{\sigma \beta} C^{\delta c} \\
& -\frac{1}{6} \varepsilon^{b c} A_{k b}^{*} A_{p c}^{*}(-1)^{\varepsilon_{k}} \mathcal{R}_{\beta, \imath}^{p}(A) M_{\delta \sigma}^{k l}(A)(-1)^{\varepsilon_{\beta}\left(\varepsilon_{\delta}+\varepsilon_{k}\right)} \hat{T}^{\sigma \beta} C^{\delta a} \\
& -\frac{1}{2} \bar{A}_{k}\left(N_{\beta \alpha}^{k}(A) \mathcal{F}_{\delta \sigma}^{\alpha}(A)+N_{\beta \sigma, \imath}^{k}(A) \mathcal{R}_{\delta}^{\imath}(A)\right) \hat{T}^{\sigma \beta} C^{\delta a}(-1)^{\varepsilon_{\delta}\left(\varepsilon_{\beta}+\varepsilon_{\sigma}\right)} \\
& +\frac{1}{4}\left(2 \bar{C}_{\gamma b}-B_{\gamma b}^{*}\right)\left[\left(\mathcal{F}_{\sigma \alpha}^{\gamma}(A) \mathcal{F}_{\delta \beta}^{\alpha}(A)\right.\right. \\
& \left.\left.+2 \mathcal{F}_{\sigma \delta, \imath}^{\gamma}(A) \mathcal{R}_{\beta}^{\imath}(A)\right)(-1)^{\varepsilon_{\sigma} \varepsilon_{\delta}}-\mathcal{F}_{\delta \alpha}^{\gamma}(A) \mathcal{F}_{\sigma \beta}^{\alpha}(A)\right] T^{\beta \sigma\{a b\}} B^{\delta} \\
& +\frac{1}{4} \varepsilon^{a b}\left(2 \bar{C}_{\gamma b}-B_{\gamma b}^{*}\right)\left[\mathcal{F}_{\sigma \alpha}^{\gamma}(A) \mathcal{F}_{\delta \beta}^{\alpha}(A)\right. \\
& \left.+2 \mathcal{F}_{\sigma \delta, \imath}^{\gamma}(A) \mathcal{R}_{\beta}^{\imath}(A)\right](-1)^{\varepsilon_{\sigma} \varepsilon_{\delta}} \hat{T}^{\beta \sigma} B^{\delta} \\
& +\frac{1}{4} A_{p b}^{*} \bar{A}_{k}\left[\left(M_{\sigma \alpha}^{p k}(A) \mathcal{F}_{\delta \beta}^{\alpha}(A)+2 M_{\sigma \delta, \imath}^{p k}(A) \mathcal{R}_{\beta}^{\imath}(A)\right.\right. \\
& \left.+2 \mathcal{R}_{\sigma, \imath}^{p}(A) M_{\delta \beta}^{k l}(A)(-1)^{\varepsilon_{k}\left(\varepsilon_{\sigma}+\varepsilon_{p}\right)}\right)(-1)^{\varepsilon_{\sigma} \varepsilon_{\delta}} \\
& \left.-M_{\delta \alpha}^{p k}(A) \mathcal{F}_{\sigma \beta}^{\alpha}(A)\right] T^{\beta \sigma\{a b\}} B^{\delta}+\frac{1}{4} \varepsilon^{a b} A_{p b}^{*} \bar{A}_{k}\left(M_{\sigma \alpha}^{p k}(A) \mathcal{F}_{\delta \beta}^{\alpha}(A)\right. \\
& +2 \mathcal{R}_{\sigma, \imath}^{p}(A) M_{\delta \beta}^{k \imath}(A)(-1)^{\varepsilon_{k}\left(\varepsilon_{\sigma}+\varepsilon_{p}\right)} \\
& \left.+2 M_{\sigma \delta, \imath}^{p k}(A) \mathcal{R}_{\beta}^{\iota}(A)\right)(-1)^{\varepsilon_{\sigma} \varepsilon_{\delta}} \hat{T}^{\beta \sigma} B^{\delta} \\
& -\frac{1}{2} \bar{A}_{p} \bar{A}_{k} \mathcal{R}_{\sigma, l}^{k}(A) M_{\delta \beta}^{p l}(A) C^{\beta a} B^{\delta} B^{\sigma}(-1)^{\varepsilon_{\sigma}\left(\varepsilon_{p}+1\right)+\varepsilon_{\delta}},
\end{aligned}
$$

Solution to Eqs. (3.11) with the right-hand side (3.32) is sought in a way similar to the one given in the case of $S_{2}$, that is, by consideration of each summond present in $F_{3}^{a}$. To this end, it is necessary to employ all the 
gauge algebra structural relations (3.1), (3.14), (3.27), (3.30), as well as the properties of structural coeffitients.

The functional $N_{\alpha \beta}^{\imath}(A)$, not being a structural coeffitient, along with the functional $N_{\alpha \beta, j}^{\imath}(A)$ are treated with the help of the differential consequence of the property (3.1)

$$
\mathcal{L}_{{ }_{\jmath} k}(A) \mathcal{R}_{\delta}^{k}(A)(-1)^{\varepsilon_{\jmath} \varepsilon_{\delta}}=-\mathcal{L}_{, k}(A) \mathcal{R}_{\delta, \jmath}^{k}(A) .
$$

Omitting details of cumbersome algebraic calculations, we give here the resultant form for $S_{3}$

$$
\begin{aligned}
S_{3}= & -\frac{1}{6} C_{\gamma a b}^{*} A_{\jmath c}^{*} Q_{\beta \delta \sigma}^{\gamma \jmath}(A) T^{\sigma \delta \beta\{a b c\}}(-1)^{\varepsilon_{\beta} \varepsilon_{\sigma}} \\
& -\frac{1}{6} C_{\gamma a b}^{*} \bar{A}_{\jmath} Q_{\beta \delta \sigma}^{\gamma \jmath}(A) T^{\beta \sigma\{a b\}} B^{\delta}(-1)^{\varepsilon_{\beta} \varepsilon_{\delta}} \\
& +\frac{1}{36} A_{\jmath b}^{*} A_{\imath c}^{*} A_{k a}^{*} \mathcal{D}_{\beta \delta \sigma}^{\jmath k}(A) T^{\sigma \delta \beta\{a b c\}}(-1)^{\varepsilon_{\beta}\left(\varepsilon_{\sigma}+\varepsilon_{\delta}\right)+\varepsilon_{\imath}} \\
& -\frac{1}{6}\left(2 \bar{C}_{\gamma b}-B_{\gamma b}^{*}\right) A_{\jmath c}^{*} Q_{\beta \delta \sigma}^{\gamma \jmath}(A) T^{\beta \sigma\{c b\}} B^{\delta}(-1)^{\varepsilon_{\beta} \varepsilon_{\delta}+\varepsilon_{\gamma}} \\
& -\frac{1}{6}\left(2 \bar{C}_{\gamma b}-B_{\gamma b}^{*}\right)\left[\mathcal{F}_{\delta \alpha}^{\gamma}(A) \mathcal{F}_{\beta \sigma}^{\alpha}(A)\right. \\
& \left.+2 \mathcal{F}_{\delta \beta, \imath}^{\gamma}(A) \mathcal{R}_{\sigma}^{\imath}(A)\right](-1)^{\varepsilon_{\beta} \varepsilon_{\delta}} \hat{T}^{\sigma \delta} C^{\beta b} \\
& +\frac{1}{12} A_{\jmath b}^{*} A_{p c}^{*} \bar{A}_{k} \mathcal{D}_{\beta \delta \sigma}^{j p k}(A) T^{\beta \sigma\{b c\}} B^{\delta}(-1)^{\varepsilon_{\beta} \varepsilon_{\delta}+\varepsilon_{\jmath}} \\
& +\bar{A}_{p} \bar{A}_{k} \mathcal{R}_{\sigma, l}^{k}(A) M_{\delta \beta}^{p \imath}(A)(-1)^{\varepsilon_{\sigma}\left(\varepsilon_{p}+\varepsilon_{\delta}\right)} \hat{T}^{\beta \sigma} B^{\delta} \\
& +\frac{1}{6} A_{p b}^{*} \bar{A}_{k}\left(2 \mathcal{R}_{\beta, \imath}^{p}(A) M_{\delta \sigma}^{k \imath}(A)(-1)^{\varepsilon_{k}\left(\varepsilon_{\beta}+\varepsilon_{p}\right)}\right. \\
& +4 \mathcal{R}_{\beta, \imath}^{k}(A) M_{\delta \sigma}^{p \imath}(A)(-1)^{\varepsilon_{p} \varepsilon_{\beta}}-M_{\beta \alpha}^{p k}(A) \mathcal{F}_{\delta \sigma}^{\alpha}(A) \\
& \left.-2 M_{\beta \delta, \imath}^{p k}(A) \mathcal{R}_{\sigma}^{\imath}(A)\right) \hat{T}^{\sigma \beta} C^{\delta b}(-1)^{\varepsilon_{\beta} \varepsilon_{\delta}} .
\end{aligned}
$$

Thus, in both the standard and $\operatorname{Sp}(2)$-covariant formulations of Lagrangian BRST quantization, the generating equations give rise to the gauge algebra structural relations for every order in power series of ghost and auxiliary fields $C^{\alpha a}, B^{\alpha}$. The boson functional

$$
\begin{aligned}
S^{(0)}= & \mathcal{L}(A)+S_{1}\left(\Phi^{A}, \Phi_{A a}^{*}, \bar{\Phi}_{A}\right)+S_{2}\left(\Phi^{A}, \Phi_{A a}^{*}, \bar{\Phi}_{A}\right) \\
& +S_{3}\left(\Phi^{A}, \Phi_{A a}^{*}, \bar{\Phi}_{A}\right)+O\left(C^{4-m} B^{m}\right) \\
& m=0,1,2,3,4
\end{aligned}
$$


satisfies the generating equations (3.8) up to the third order inclusively. Since arbitrariness in solution to Eqs. (3.8) is completely described in Refs. 15,17 , we omit the discussion of this question.

Consider a particular case of gauge theories of rank 1 with a closed algebra, i. e. let

$$
M_{\delta \beta}^{p \imath}(A)=Q_{\beta \delta \sigma}^{\gamma \jmath}(A)=D_{\beta \delta \sigma}^{\gamma}(A)=\mathcal{D}_{\beta \delta \sigma}^{\jmath \imath k}(A)=0
$$

Then, the functional (3.34) takes on the form

$$
S^{(0)}\left(\Phi^{A}, \Phi_{A a}^{*}, \bar{\Phi}_{A}\right)=\mathcal{L}(A)+\Phi_{A a}^{*} X^{A a}+\bar{\Phi}_{A} Y^{A}
$$

where

$$
X^{A a}=\left(X_{1}^{\imath a}, X_{2}^{\alpha a}, X_{3}^{\alpha a b}\right), Y^{A}=\left(Y_{1}^{\imath}, Y_{2}^{\alpha}, Y_{3}^{\alpha a}\right)
$$

given this

$$
\begin{aligned}
X_{1}^{\imath a}= & \mathcal{R}_{\alpha}^{\imath}(A) C^{\alpha a} \\
X_{2}^{\alpha a}= & -\frac{1}{2} \mathcal{F}_{\gamma \beta}^{\alpha}(A) B^{\beta} C^{\gamma a}-\frac{1}{12}(-1)^{\varepsilon_{\beta}}\left(\mathcal{F}_{\gamma \sigma}^{\alpha}(A) \mathcal{F}_{\beta \rho}^{\sigma}(A)\right. \\
& \left.+2 \mathcal{F}_{\gamma \beta, \imath}^{\alpha}(A) \mathcal{R}_{\rho}^{\imath}(A)\right) C^{\rho b} C^{\beta a} C^{\gamma c} \varepsilon_{c b}, \\
X_{3}^{\alpha a b}= & -\varepsilon^{a b} B^{\alpha}-\frac{1}{2}(-1)^{\varepsilon_{\beta}} \mathcal{F}_{\beta \gamma}^{\alpha}(A) C^{\gamma b} C^{\beta a}, \\
Y_{1}^{\imath}= & \mathcal{R}_{\alpha}^{\imath}(A) B^{\alpha}+\frac{1}{2}(-1)^{\varepsilon_{\alpha}} \mathcal{R}_{\alpha, \jmath}^{\imath}(A) \mathcal{R}_{\beta}^{\jmath}(A) C^{\beta b} C^{\alpha a} \varepsilon_{a b}, \\
Y_{2}^{\alpha}= & 0, Y_{3}^{\alpha a}=-2 X_{2}^{\alpha a} .
\end{aligned}
$$

The result (3.36), (3.37) coincides with the exact solution to the generating equations for $S^{(0)}$ obtained in Ref. 15 in the case of gauge theories of rank 1 with a closed algebra.

\section{Sp(2)-covariant Quantization of the Yang-Mills Theory}

It may not be out of place to illustrate general results and relations of this paper on a basis of a simple example of a concrete gauge theory. To do 
this we consider the Yang-Mills theory described by the action

$$
\mathcal{L}=\mathcal{L}\left(A^{\mu m}\right)=-\frac{1}{4} \int d^{4} x G_{\mu \nu}^{m} G^{\mu \nu m} .
$$

The Yang-Mills field $A^{\mu m}(x)$ is defined on the Minkovsky space and assumes its values in the adjoint representation of a semisimple compact group. The field strength $G_{\mu \nu}^{m}$ has the form

$$
G_{\mu \nu}^{m}=\partial_{\mu} A_{\nu}^{m}-\partial_{\nu} A_{\mu}^{m}+f^{m n l} A_{\mu}^{n} A_{\nu}^{l}
$$

where the Greek subscripts $\mu, \nu$ refer to the Minkovsky space, while the Roman subscripts $k, l, m, n, p$, refer to the internal symmetry group indices. The Yang-Mills fields $A^{\mu m}$ play the role of initial fields $A^{\imath}$. The action (4.1) is invariant under the gauge transformations

$$
\delta A_{\mu}^{m}(x)=\mathcal{D}_{\mu}^{m n}(x) \xi^{n}(x)=\int d^{4} y \mathcal{R}_{\mu}^{m n}(x ; y) \xi^{n}(y)
$$

where

$$
\mathcal{D}_{\mu}^{m n}=\delta^{m n} \partial_{\mu}+f^{m l n} A_{\mu}^{l}
$$

is the covariant derivative and $\mathcal{R}_{\mu}^{m n}(x ; y)$ are the generators of the gauge transformations

$$
\mathcal{R}_{\mu}^{m n}(x ; y)=\mathcal{D}_{\mu}^{m n} \delta(x-y) .
$$

In (4.2) $f^{l m n}$ are the structural constants associated with the symmetry group (the interaction constant is absorbed into $f^{l m n}$ ), $\xi^{n}(y)$ are arbitrary functions. The condensed indices $\imath, \alpha$ for the theory in question are understood in the form

$$
\imath=(\mu, m, x), \quad \alpha=(m, x) .
$$

The structural coeffitients $\mathcal{F}_{\alpha \beta}^{\gamma}$ arising in the relations (3.14) are defined by the group structural constants $f^{l m n}$ in the form $(\alpha=(m, x), \beta=(n, y), \gamma=$ $(l, z))$

$$
\mathcal{F}_{\alpha \beta}^{\gamma}=f^{l m n} \delta(x-z) \delta(y-z)
$$


while $M_{\alpha \beta}^{\imath \jmath}=0$. The higher gauge algebra structural coeffitients $Q_{\beta \gamma \delta}^{\imath \alpha}, \mathcal{D}_{\beta \gamma \delta}^{\imath \jmath k}$ also assume zero value. The total configuration space of the theory is defined by the set of fields

$$
\Phi^{A}=\left(A^{\mu m}, B^{m}, C^{m a}\right)
$$

and therefore, the set of antifields $\Phi_{A a}^{*}$ and $\bar{\Phi}_{A}$ have the form

$$
\Phi_{A a}^{*}=\left(A_{\mu a}^{* m}, B_{a}^{* m}, C_{a b}^{* m}\right), \quad \bar{\Phi}_{A}=\left(\bar{A}_{\mu}^{m}, \bar{B}^{m}, \bar{C}_{a}^{m}\right) .
$$

Given this, the Grassmann parity and the new ghost number assume the values $\left(\varepsilon_{i}=\varepsilon_{\alpha}=0\right)$

$$
\begin{gathered}
\varepsilon\left(A^{\mu m}\right)=\varepsilon\left(B^{m}\right)=0, \quad \varepsilon\left(C^{m a}\right)=1, \\
\varepsilon\left(A_{\mu_{a}}^{* m}\right)=\varepsilon\left(B_{a}^{* m}\right)=1, \quad \varepsilon\left(C_{a b}^{* m}\right)=0, \\
\varepsilon\left(\bar{A}_{\mu}^{m}\right)=\varepsilon\left(\bar{B}^{m}\right)=0, \quad \varepsilon\left(\bar{C}_{a}^{m}\right)=1, \\
\operatorname{ngh}\left(A^{\mu m}\right)=0, \quad \operatorname{ngh}\left(C^{a m}\right)=1, \quad \operatorname{ngh}\left(B^{m}\right)=2, \\
\operatorname{ngh}\left(A_{\mu a}^{* m}\right)=-1, \quad \operatorname{ngh}\left(C_{a b}^{* m}\right)=-2, \quad \operatorname{ngh}\left(B_{a}^{* m}\right)=-3, \\
\operatorname{ngh}\left(\bar{A}_{\mu}^{m}\right)=-2, \quad \operatorname{ngh}\left(\bar{C}_{a}^{m}\right)=-3, \quad \operatorname{ngh}\left(\bar{B}^{m}\right)=-4 .
\end{gathered}
$$

By virtue of the manifest structure (4.1)-(4.3) of the theory in question, the solutions to the generating equations for $S_{1}, S_{2}, S_{3}$ are representable in the following local form

$$
\begin{aligned}
S_{1}= & \int d^{4} x\left\{A_{\mu a}^{* m} \mathcal{D}^{\mu m n} C^{n a}+\bar{A}_{\mu}^{m} \mathcal{D}^{\mu m n} B^{n}-C_{a b}^{* m} B^{m} \varepsilon^{a b}\right\}, \\
S_{2}= & \int d^{4} x\left\{\left(\bar{C}_{a}^{m}-\frac{1}{2} B_{a}^{* m}\right) f^{m n l} B^{l} C^{n a}\right. \\
& \left.\quad-\frac{1}{2} C_{a b}^{* m} f^{m n l} C^{l b} C^{n a}-\frac{1}{2} \bar{A}_{\mu}^{m} f^{m n l} C^{n a} \mathcal{D}^{\mu l k} C^{k b} \varepsilon_{a b}\right\}, \\
S_{3}=- & \frac{1}{12} \int d^{4} x\left\{\left(B_{a}^{* m}-2 \bar{C}_{a}^{m}\right) f^{m n l} f^{l k p} C^{p b} C^{k a} C^{n c} \varepsilon_{c b}\right\} .
\end{aligned}
$$

Since the Yang-Mills theory belongs to the theories of rank 1, the functional $S=\mathcal{L}+S_{1}+S_{2}+S_{3}$ is an exact solution to the generating equations. 
Moreover, the antisymmetry properties of the structural constants $f^{l m n}$ lead to the fact that the functional $S$ is also a solution to Eqs. (3.3).

Consider for the theory in question the generating functional of Green's functions $Z(J)$ (3.6). Choose the gauge functional $F$ in the form

$$
F=-\frac{\alpha}{2} \int d^{4} x A_{\mu}^{m} A^{\mu m}
$$

Then, integrating in the functional integral (3.6) over the variables $\lambda^{A}$, $\Pi^{A a}, \bar{\Phi}_{A}, \Phi_{A a}^{*}$ and taking (4.4), (4.5) into account, we obtain the following representation for the generating functional $Z(J)$

$$
Z(J)=\int d \Phi \exp \left\{\frac{i}{\hbar}\left(\mathcal{L}+S_{\mathrm{GH}}(\Phi)+S_{\mathrm{GF}}(\Phi)+J_{A} \Phi^{A}\right)\right\},
$$

where the following notations are used

$$
\begin{gathered}
S_{\mathrm{GH}}=\frac{\alpha}{2} \int d^{4} x \partial^{\mu} C^{m a} \mathcal{D}_{\mu}^{m n} C^{n b} \varepsilon_{a b}, \\
S_{\mathrm{GF}}=\alpha \int d^{4} x B^{m} \partial^{\mu} A_{\mu}^{m} .
\end{gathered}
$$

Note that the integrand in Eq. (4.6) for $J_{A}=0$ is invariant under the extended BRST symmetry transformations of the form

$$
\begin{gathered}
\delta A_{\mu}^{m}=\mathcal{D}_{\mu}^{m n} C^{n a} \mu_{a}, \\
\delta B^{m}=-\frac{1}{2}\left(f^{m n l} B^{l} C^{n a}+\frac{1}{6} f^{m n l} f^{l k p} C^{p b} C^{k a} C^{n c} \varepsilon_{c b}\right) \mu_{a}, \\
\delta C^{m a}=\left(\varepsilon^{a b} B^{m}-\frac{1}{2} f^{m n l} C^{l a} C^{n b}\right) \mu_{b} .
\end{gathered}
$$

Now consider the Yang-Mills theory in the Hamiltonian version of $\operatorname{Sp}(2)$ covariant quantization. Note to this end that the corresponding dynamical system is described in the initial phase space $\eta\left(x^{\mu}=\left(x^{0}, \vec{x}\right)\right.$, the spatial indices are denoted $i, j: \mu=(0, i))$

$$
\eta=\left(p_{\imath}, q^{\imath}\right)=\left(\Pi_{i}^{m}, A^{i m}\right), \quad \imath=(i, m, \vec{x}), \quad \varepsilon\left(A^{i m}\right)=0
$$

by the classical Hamiltonian $H_{0}$

$$
H_{0}=\int d^{3} x\left\{-\frac{1}{2} \Pi_{i}^{m} \Pi^{i m}+\frac{1}{4} F_{i j}^{m} F^{i j m}\right\}
$$


and by the set of lineary independent constraints $T_{\alpha}(\alpha=(m, \vec{x}))$

$$
T_{\alpha}=T^{m} \equiv \mathcal{D}_{i}^{m n} \Pi^{i n}
$$

with the following involution relaitons

$$
\begin{gathered}
\left\{T^{m}(x), T^{n}(y)\right\}=\int d^{3} z f^{l m n} T^{l}(z) \delta(\vec{x}-\vec{z}) \delta(\vec{y}-\vec{z}), \quad x^{0}=y^{0}=z^{0}, \\
\left\{T^{m}(x), H_{0}\right\}=0 .
\end{gathered}
$$

Hence the structural coeffitients $U_{\alpha \beta}^{\gamma}$, arising in Eqs. (2.1) have the form $(\alpha=(m, \vec{x}), \beta=(n, \vec{y}), \gamma=(l, \vec{z}))$

$$
U_{\alpha \beta}^{\gamma}=f^{l m n} \delta(\vec{x}-\vec{z}) \delta(\vec{y}-\vec{z}),
$$

whereas $V_{\alpha}^{\beta}=0$. Given this, the higher gauge algebra structural coeffitients $E_{\alpha \beta}^{\gamma \delta}, E_{\alpha \beta \gamma}^{\delta \rho}, E_{\alpha \beta \gamma}^{\delta \rho \sigma}$ are equal to zero.

The extended phase space $\Gamma$ for the dynamical system in question has the form

$$
\Gamma=\left(P_{A}, Q^{A}\right)=\left(\eta ; \mathcal{P}_{a}^{m}, C^{m a} ; \lambda^{m}, \pi^{m}\right)
$$

where the Grassmann parity and the new ghost number of the variables belong to $\Gamma$ are as follows

$$
\begin{gathered}
\varepsilon\left(C^{m a}\right)=1, \quad \varepsilon\left(\pi^{m}\right)=0, \\
\operatorname{ngh}\left(C^{m a}\right)=1, \quad \operatorname{ngh}\left(\pi^{m}\right)=2 .
\end{gathered}
$$

The explicit form of the gauge algebra structural coeffitients (4.7) and the manifest structure of the extended phase space $\Gamma$ (4.8) enable us, with allowance made for Eqs. (2.44), to give solutions to the generating equations for $\mathcal{H}, \Omega^{a}$

$$
\mathcal{H}=H_{0},
$$




$$
\begin{aligned}
\Omega^{a}= & \int d^{3} x\left\{C^{m a} \mathcal{D}_{i}^{m n} \Pi^{i n}+\varepsilon^{a b} \mathcal{P}_{b}^{m} \pi^{m}+\frac{1}{2} \mathcal{P}_{b}^{l} f^{l m n} C^{n a} C^{m b}\right. \\
& \left.-\frac{1}{2} \lambda^{l} f^{l m n} C^{n a} \pi^{m}-\frac{1}{12} \lambda^{l} f^{l m n} f^{n k p} C^{p a} C^{k b} C^{m c} \varepsilon_{b c}\right\} .
\end{aligned}
$$

Now consider, by virtue of (4.9), the generating functional of Green's functions $Z(I)$ (2.8) in the Hamiltonian version of $\mathrm{Sp}(2)$-covariant quantization. To this end we choose the boson gauge function $\Phi$ in Eq. (2.6), determining the unitarizing Hamiltonian $H$, in the form

$$
\Phi=\int d^{3} x\left\{\frac{\alpha}{2} A_{i}^{m} A^{i m}-\frac{1}{2 \alpha} \lambda^{m} \lambda^{m}\right\} .
$$

Then, integrating in the functional integral (2.8) over the momenta $\mathcal{P}_{a}^{m}$ and assuming the corresponding sources to be equal to zero, we obtain, with allowance made for the notations of the form

$$
A_{0}^{m} \equiv-\alpha^{-1} \lambda^{m}, \quad B^{m} \equiv \pi^{m},
$$

the following representation for the generating functional of Green's functions $(2.8)$

$$
Z(J)=\int d \Phi \exp \left\{\frac{i}{\hbar}\left(\mathcal{L}+S_{\mathrm{GH}}+S_{\mathrm{GF}}+J_{A} \Phi^{A}\right)\right\} .
$$

Here $\Phi^{A}$ and $J_{A}$ coincide with the sets of fields of the total configuration space (4.3) and the corresponding sources respectively. The functional $\mathcal{L}$ is the classical action (4.1), whereas the functionals $S_{\mathrm{GH}}, S_{\mathrm{GF}}$ are defined in Eq. (4.6). Hence we conclude that for the fields of the total configuration space in the theory (4.1)-(4.2), the generating functionals of Green's functions of both the Lagrangian and Hamiltonian versions of $\operatorname{Sp}(2)$-covariant quantization coincide.

\section{Acknowledgment}

The authors are grateful to I. A. Batalin and I. V. Tyutin for useful discussions. The work is supported in part by the International Science Foundation, grant RI 1000 and by the Russian Foundation for Fundamental Research, project No. 94-02-03234. 


\section{References}

1. B. de Wit and J. W. van Holten, Phys. Lett. B79 (1978) 389.

2. I. A. Batalin and G. A. Vilkovisky, Phys. Lett. B102 (1981) 27.

3. I. A. Batalin and G. A. Vilkovisky, Phys. Rev. D28 (1983) 2576.

4. E. S. Fradkin and G. A. Vilkovisky, Phys. Lett. B55 (1975) 224.

5. I. A. Batalin and G. A. Vilkovisky, Phys. Lett. B69 (1977) 309.

6. E. S. Fradkin and T. E. Fradkina, Phys. Lett. B72 (1978) 343.

7. C. Becchi, A. Rouet and R. Stora, Commun. Math. Phys. 42 (1975) 127.

8. I. V. Tyutin, preprint Lebedev Inst. No. 39, 1975.

9. N. Nakanishi and I. Ojima, Z. Phys. C6 (1980) 255.

10. L. Alvarez-Gaume and L. Baulieu, Nucl. Phys. B212 (1983) 255.

11. S. Hwang, Nucl. Phys. B231 (1984) 386.

12. V. P. Spiridonov, Nucl. Phys. B308 (1988) 527.

13. G. Curci and R. Ferrari, Phys. Lett. B63 (1976) 91.

14. I. Ojima, Progr. Theor. Phys. 63 (1979) 625.

15. I. A. Batalin, P. M. Lavrov and I. V. Tyutin, J. Math. Phys. 31 (1990) 1487.

16. I. A. Batalin, P. M. Lavrov and I. V. Tyutin, J. Math. Phys. 32 (1991) 532.

17. I. A. Batalin, P. M. Lavrov and I. V. Tyutin, J. Math. Phys. 32 (1991) 2513. 
18. I. A. Batalin, P. M. Lavrov and I. V. Tyutin, J. Math. Phys. 31 (1990) 6.

19. I. A. Batalin, P. M. Lavrov and I. V. Tyutin, J. Math. Phys. 31 (1991) 2708.

20. I. A. Batalin, P. M. Lavrov and I. V. Tyutin, Int. J. Mod. Phys. A6 (1991) 3599.

21. B. S. De Witt, Dynamical Theory of Groups and Fields (Gordon and Breach, New York, 1965).

22. E. S. Fradkin, proceedings of the X Winter School of Theoretical Physics in Karpacs, No. 207, 1973.

23. I. A. Batalin and G. A. Vilkovisky, J. Math. Phys. 26 (1985) 172. 